\title{
REDUCED DYNAMICS AND LAGRANGIAN SUBMANIFOLDS OF SYMPLECTIC MANIFOLDS
}

\author{
E. GARCÍA-TORAÑO ANDRÉS, E. GUZMÁN, J.C. MARRERO, AND T. MESTDAG
}

\begin{abstract}
In this paper, we will see that the symplectic creed by Weinstein "everything is a Lagrangian submanifold" also holds for Hamilton-Poincaré and Lagrange-Poincaré reduction. In fact, we show that solutions of the Hamilton-Poincaré equations and of the Lagrange-Poincaré equations are in one-to-one correspondence with distinguished curves in a Lagrangian submanifold of a symplectic manifold. For this purpose, we will combine the concept of a Tulczyjew triple with Marsden-Weinstein symplectic reduction.
\end{abstract}

1. Introduction

2. Tulczyjew's triple and Marsden-Weinstein reduction

3. Reduced Lagrangian submanifolds

4. Hamilton-Poincaré reduction

5. Special Cases

5.1. The case where the configuration space is a Lie group

5.2. The case where the configuration space is a product

6. Lagrange-Poincaré reduction

7. The equivalence between the reduced Lagrangian and Hamiltonian formalism

8. Conclusions and future work

References

Contents

\section{INTRODUCTION}

Lagrangian and Hamiltonian mechanics can both be formulated in the context of symplectic geometry. For a Hamiltonian system, one may simply consider the canonical symplectic form on the cotangent bundle of the configuration space. For the case of a Lagrangian system, the regularity of the Lagrangian plays a role: if the Lagrangian is (hyper)regular, one may use the Legendre transformation to pull back the canonical symplectic form to obtain a symplectic form on the tangent bundle, the so-called Poincaré-Cartan two-form. Less known is that even in the case when the Lagrangian is singular, there exist symplectic formulations of the dynamics. One such formulation is provided by the so-called Tulczyjew triple [23, 24], which consists of three (anti)symplectomorphic manifolds. We will provide all details when needed, but, briefly speaking, it describes the dynamics in terms of Lagrangian submanifolds of the spaces of the triple, and it ultimately provides

2010 Mathematics Subject Classification. 53D05, 53D12, 70G65, 70H03, 70H05, 70H33.

Key words and phrases. Marsden-Weinstein reduction, Tulczyjew's triple, Lagrangian submanifold, Hamilton-Poincaré equations, Lagrange-Poincaré equations.

E. Guzmán and J. C. Marrero acknowledge support from MEC (Spain) Grants MTM2011-15725-E, MTM2012-34478, the project of the Canary Government ProdID20100210 and the European Community IRSES-project GEOMECH-246981. E. Guzmán also wishes to thank the CSIC for a JAE-predoc grant. E. García-Toraño Andrés and T. Mestdag acknowledge support from the Research Foundation - Flanders (FWO). 
a unified picture where both Hamiltonian and Lagrangian mechanics can be treated on the same footing (see [23, 24] and Section 21). The ideas behind this triple have been extended to more general structures (such as Lie algebroids [9] or Dirac structures [8]) and to more general classes of systems (such as systems with constraints [7], time dependent systems [10] and field theories [2], 6], [15]).

Reduction theories provide a way to benefit from symmetry properties of dynamical systems. One such theory is that of Lagrange-Poincaré reduction which, in a few words, uses the symmetry group of the dynamics to reduce Hamilton's principle. The Hamiltonian analogue of Lagrange-Poincaré reduction is Hamiltonian-Poincaré reduction. In the literature, there exist many distinct geometric models for the equations that result from this procedure, mostly for the case of a regular Lagrangian [4, 5, 14, 22. It has also been observed that the Lagrange-Poincaré equations may be considered as Euler-Lagrange equations on a Lie algebroid, for the case of the so-called Atiyah algebroid [9, 14]. One of the objectives of this paper is to provide a new framework in which also the case of singular Lagrangians can be included. In [5] Lagrange-Poincaré reduction and Hamilton-Poincaré reduction is said to be "outside the realm of symplectic (and Routh) reduction". The main goal of this paper is to show Tulczyjew's ideas concerning dynamics on the one hand and symplectic reduction on the other hand can be combined to a model for LagrangePoincaré reduction and Hamilton-Poincaré reduction within a reduced Tulczyjew triple. The core idea behind the new triple is that it is purely composed of symplectic manifolds, as was its unreduced version. To do so, we will need to discuss first the reduction (via the Marsden-Weinstein procedure) of an invariant Lagrangian submanifold. Then, we will describe Hamilton-Poincaré and Lagrange-Poincaré equations in terms of Lagrangian submanifolds of symplectic manifolds. So, we may conclude that the symplectic creed as formulated by Weinstein [25] in the form "everything is a Lagrangian submanifold" also holds in this theory.

In the literature one may find three seemingly related approaches. In [9] the authors obtain a Tulczyjew triple in a Lie algebroid setting. If one applies these results to the case when the Lie algebroid is the Atiyah algebroid, one obtains rather a Poisson answer than a symplectic one. We will relate our approach to theirs in the last section. In a second approach [14] one may find a different Tulczyjew triple for Lie algebroids. This triple consists of so-called prolongation bundles of Lie algebroids, which are all so-called 'symplectic Lie algebroids'. The concept of a symplectic Lie algebroid is a generalization of a symplectic manifold to the level of a vector bundle, but not a genuine symplectic manifold in its own right. A third approach (in e.g. [26, 27]) also deals with singular Lagrangian systems, but within the context of Dirac structures.

The paper is structured as follows. In Section 2, we recall some basic results on Tulczyjew's triple and on Marsden-Weinstein symplectic reduction. In Section 3, we show that an invariant Lagrangian submanifold of a symplectic manifold endowed with a Hamiltonian action may, under additional assumptions, be reduced to a Lagrangian submanifold of the reduced symplectic manifold. In Section 4, we give a one-to-one correspondence between solutions of the Hamilton-Poincaré equations on the one hand, and distinguished curves in a Lagrangian submanifold of a symplectic manifold on the other hand. In Section 5. we discuss two interesting special cases: the case where the configuration space is the symmetry Lie group and the case where the configuration space is the product of the symmetry Lie group with a base manifold. In Section [6, we prove that there exists a 
one-to-one correspondence between solutions of the Lagrange-Poincaré equations and distinguished curves in a Lagrangian submanifold of the same symplectic manifold as in the Hamiltonian side. Finally, in Section 7, we show that, for a hyperregular Lagrangian, the corresponding Lagrangian submanifolds in the Lagrangian and Hamiltonian side coincide. The paper ends with our conclusions and with some directions for future research.

\section{TulczyjeW's triple and Marsden-Weinstein ReduCtion}

We explain the main characteristics of the Tulczyjew triple in some detail. Let $Q$ be the configuration manifold of a mechanical system. A Lagrangian function $L$ on $T Q$ defines a submanifold $d L(T Q)$, which is Lagrangian with respect to the canonical symplectic structure $\omega_{T Q}$ on $T^{*} T Q$.

This submanifold can be mapped into $T T^{*} Q$ via the inverse of Tulczyjew's diffeomorphism

$$
\begin{aligned}
A_{Q}: T T^{*} Q & \rightarrow T^{*} T Q, \\
(q, p, \dot{q}, \dot{p}) & \mapsto(q, \dot{q}, \dot{p}, p) .
\end{aligned}
$$

This map is a symplectomorphism when we consider on $T T^{*} Q$ the symplectic structure $\omega_{Q}^{c}$, which is given by the complete lift of the canonical symplectic form $\omega_{Q}$ on $T^{*} Q$. Therefore, also $S_{L}=A_{Q}^{-1}(d L(T Q))$ is a Lagrangian submanifold. In [23, 24], it is shown that solutions of the Euler-Lagrange equations are in one-to-one correspondence with curves in $S_{L}$ which are tangent lifts of curves in $T^{*} Q$.

In the Hamiltonian formulation it is possible to proceed in a similar way. Here, the Lagrangian submanifold $d H\left(T^{*} Q\right)$ of $\left(T^{*} T^{*} Q, \omega_{T^{*} Q}\right)$ may be mapped into $T T^{*} Q$ via the isomorphism vector bundle

$$
\begin{aligned}
b_{\omega_{Q}}: T T^{*} Q & \rightarrow T^{*} T^{*} Q, \\
(q, p, \dot{q}, \dot{p}) & \mapsto(q, p,-\dot{p}, \dot{q}),
\end{aligned}
$$

which is induced by the symplectic form $\omega_{Q}$. Since this map is an anti-symplectomorphism, $S_{H}=b_{\omega_{Q}}^{-1}\left(d H\left(T^{*} Q\right)\right)$ is a Lagrangian submanifold of $\left(T T^{*} Q, \omega_{Q}^{c}\right)$. In fact, it is the image of the Hamiltonian vector field $X_{H}$. As in the Lagrangian case, solutions of the Hamilton equations are in one-to-one correspondence with curves in $S_{H}$ which are tangent lifts of curves in $T^{*} Q$.

The following diagram, which is known as Tulczyjew's triple, illustrates the previous situation

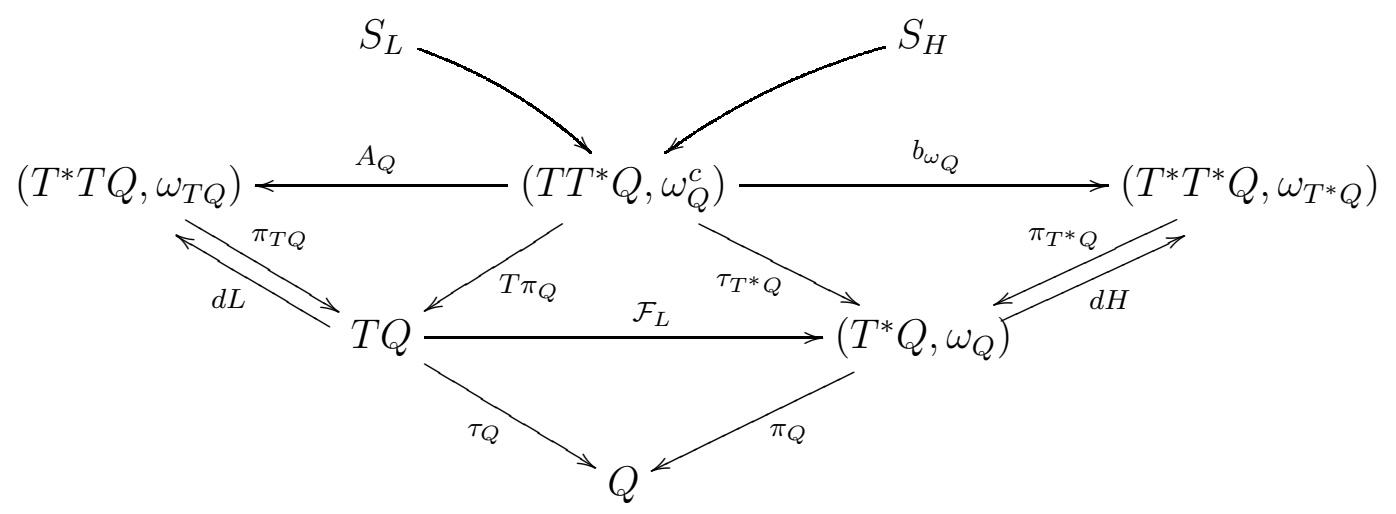

Throughout the next sections, we shall often apply the Marsden-Weinstein reduction theorem. For completeness, we now give a concise outline of this technique. This will 
allow us to fix the notations used in the rest of the paper. For a detailed treatment of this topic, see [1, 20].

It is well known that if a Lie group $G$ acts freely and properly on a manifold $M$, the space of orbits $M / G$ is a smooth manifold and $M$ is the total space of a principal $G$-bundle with bundle projection $p_{M}: M \rightarrow M / G$.

An action $\phi: G \times M \rightarrow M$ of a Lie group $G$ on a symplectic manifold $(M, \omega)$ is called $G$-Hamiltonian if for each $g \in G$ the map $\phi_{g}: M \rightarrow M$ is a symplectomorphism (i.e. $\left.\phi_{g}^{*} \omega=\omega\right)$ and $\phi$ admits an $A d^{*}$-equivariant momentum map $J: M \rightarrow \mathfrak{g}^{*}$. Here $A d^{*}$ equivariance means

$$
J\left(\phi_{g}(x)\right)=A d_{g^{-1}}^{*} J(x), \quad \text { for any } x \in M, g \in G,
$$

where $A d^{*}: G \times \mathfrak{g}^{*} \rightarrow \mathfrak{g}^{*}$ is the dual of the adjoint action. The momentum map $J$ guarantees that the infinitesimal generators $\xi_{M}$ of the action $\phi$ are globally Hamiltonian vector fields: $\xi_{M}$ becomes a Hamiltonian vector field for the Hamiltonian function $J_{\xi}: M \rightarrow \mathbb{R}$ defined as

$$
J_{\xi}(x)=\langle J(x), \xi\rangle, \quad \text { for all } x \in M \text { and } \xi \in \mathfrak{g},
$$

that is, $i_{\xi_{M}} \omega=d J_{\xi}$. Throughout the paper, unless otherwise stated, we will impose the following two assumptions:

(1) We assume that $\mu \in \mathfrak{g}^{*}$ is a regular value of the momentum map, which guarantees that $J^{-1}(\mu)$ is an embedded submanifold of $M$. If we consider the isotropy group of $\mu$ with respect to the coadjoint action,

$$
G_{\mu}=\left\{g \in G: A d_{g^{-1}}^{*} \mu=\mu\right\},
$$

one can prove that $G_{\mu}$ is a closed subgroup of $G$ which, due to the equivariance condition of the momentum map, leaves $J^{-1}(\mu)$ invariant. Thus, it makes sense to consider the $G_{\mu}$-action on $J^{-1}(\mu)$,

$$
\phi_{\mu}: G_{\mu} \times J^{-1}(\mu) \rightarrow J^{-1}(\mu) .
$$

(2) We will assume that $G_{\mu}$ acts freely and properly on $J^{-1}(\mu)$. Then, the space of orbits $J^{-1}(\mu) / G_{\mu}$ admits a manifold structure such that the canonical projection

$$
p_{J^{-1} \mu}: J^{-1}(\mu) \rightarrow J^{-1}(\mu) / G_{\mu}
$$

is the bundle projection of a principal $G_{\mu}$-bundle. The main result in [20] is that the reduced manifold $J^{-1}(\mu) / G_{\mu}$ admits a symplectic form $\omega_{\mu}$ characterized by the equation $p_{J^{-1}(\mu)}^{*} \omega_{\mu}=i^{*} \omega$, where $i: J^{-1}(\mu) \rightarrow M$ is the canonical inclusion.

Remark 2.1. The following observations are important in the forthcoming sections:

i) In the presence of a $G$-action $\phi$ on $M$, it is customary to simply write $g x$ for $\phi_{g}(x)$. We will use this notation when there is no risk of confusion.

ii) For the rest of the paper we will assume, unless otherwise stated, that all the actions are free and proper. Under these conditions, the assumptions on the regularity of the momentum map and on the freeness and properness of $\phi_{\mu}$ hold. Indeed in this case $J$ is a submersion and the induced action of $G_{\mu}$ on $J^{-1}(\mu)$ is free and proper, and it follows that the quotient space $M / G$ is a manifold and that $p_{M}: M \rightarrow M / G$ is the bundle projection of a principal $G$-bundle. Moreover, the connected component of $J^{-1}(\mu) / G_{\mu}$ may be identified with the symplectic leaf of the Poisson manifold $M / G$. 
Recall that the symplectic manifold $M$ induces a Poisson structure $\{., .\}_{M / G}$ on $M / G$ which is defined by

$$
\{f, h\}_{M / G} \circ p_{M}=\left\{f \circ p_{M}, h \circ p_{M}\right\}_{M}, \quad \text { for all } f, h \in C^{\infty}(M / G),
$$

where $\{., .\}_{M}$ is the Poisson bracket on $M$ defined by the symplectic structure.

iii) In what follows, we will mainly use Marsden-Weinstein reduction for the case where $\mu=0$. In that case, however, it actually coincides with coisotropic reduction (see [1]).

Example 2.2. A typical example of Marsden-Weinstein reduction is cotangent bundle reduction. A $G$-action $\phi$ on $M$ may be lifted to a $G$-action $\phi^{T^{*} M}$ on $T^{*} M$ which is given by cotangent lifts:

$$
g \alpha_{x}=\left(T_{g x}^{*} \phi_{g^{-1}}\right)\left(\alpha_{x}\right), \quad \text { for all } \alpha_{x} \in T^{*} M \text { and } g \in G .
$$

If $\phi$ is free and proper, then so is $\phi^{T^{*} M}$. This action preserves the Liouville one-form $\lambda_{M}$, and therefore it also preserves the canonical symplectic form $\omega_{M}$ of the cotangent bundle $T^{*} M$. It admits an $A d^{*}$-equivariant momentum map $J_{T^{*} M}: T^{*} M \rightarrow \mathfrak{g}^{*}$ given by

$$
\left\langle J_{T^{*} M}\left(\alpha_{x}\right), \xi\right\rangle=\left\langle\alpha_{x}, \xi_{M}(x)\right\rangle, \quad \text { for all } \alpha_{x} \in T^{*} M \text { and } \xi \in \mathfrak{g} .
$$

As the assumptions of the aforementioned reduction apply, it follows that $J_{T^{*} M}^{-1}(\mu) / G_{\mu}$ is a symplectic manifold. In [17, one finds a broad study of cotangent bundle reduction which characterizes the cases in which the reduced symplectic manifold $J_{T^{*} M}^{-1}(\mu) / G_{\mu}$ is again a cotangent bundle and in particular, it is shown that for $\mu=0$ one has an identification

$$
\left(J_{T^{*} M}^{-1}(0) / G,\left(\omega_{M}\right)_{0}\right) \cong\left(T^{*}(M / G), \omega_{M / G}\right) .
$$

The vector bundle isomorphism $\Psi_{0}: J_{T^{*} M}^{-1}(0) / G \rightarrow T^{*}(M / G)$ (over the identity in $M / G$ ) which realizes this identification is characterized by the following condition:

$$
\left\langle\Psi_{0}\left(p_{J_{T^{*} M}^{-1}(0)}\left(\alpha_{x}\right)\right),\left(T_{x} p_{M}\right)\left(v_{x}\right)\right\rangle=\left\langle\alpha_{x}, v_{x}\right\rangle,
$$

for $\alpha_{x} \in J_{T^{*} M}^{-1}(0)$ and $v_{x} \in T M$. In fact, $J_{T^{*} M}^{-1}(0)$ is identified with the annihilator $V^{\circ} p_{M}$ of the vertical bundle $V p_{M}$ of the canonical projection $p_{M}: M \rightarrow M / G$. Thus, $J_{T^{*} M}^{-1}(0) / G=V^{\circ} p_{M} / G$ and the latter space is canonically identified with $T^{*}(M / G) . \triangleleft$

Finally, we recall that if two symplectomorphic manifolds are both Marsden-Weinstein reducible for the same symmetry group and have compatible actions, then the reduced spaces are also symplectomorphism (see, for example, [12]). More specifically, let $f: M_{1} \rightarrow$ $M_{2}$ be a symplectomorphism between the symplectic manifolds $\left(M_{1}, \omega_{1}\right)$ and $\left(M_{2}, \omega_{2}\right)$ and suppose that both $M_{1}$ and $M_{2}$ admit $G$-Hamiltonian actions with $A d^{*}$-equivariant momentum maps $J_{1}$ and $J_{2}$ respectively. If $f$ is $G$-equivariant and $J_{2} \circ f=J_{1}$, then for a fixed value $\mu \in \mathfrak{g}^{*}$ it follows that the reduced manifolds $J_{1}^{-1}(\mu) / G_{\mu}$ and $J_{2}^{-1}(\mu) / G_{\mu}$ are symplectomorphic, with symplectomorphism

$$
\left[f_{\mu}\right]:\left(J_{1}^{-1}(\mu) / G_{\mu}, \omega_{1 \mu}\right) \rightarrow\left(J_{2}^{-1}(\mu) / G_{\mu}, \omega_{2 \mu}\right) .
$$

In the next lines, we will briefly explain how this map is defined. Observing that the map $f: M_{1} \rightarrow M_{2}$ preserves the momentum maps, it follows that $f\left(J_{1}^{-1}(\mu)\right)=J_{2}^{-1}(\mu)$ for each value $\mu \in \mathfrak{g}^{*}$. If we denote by $f_{\mu}: J_{1}^{-1}(\mu) \rightarrow J_{2}^{-1}(\mu)$ the restriction of the map $f$ to the submanifold $J_{1}^{-1}(\mu)$, then $f_{\mu}$ is a $G_{\mu}$-equivariant diffeomorphism (because $f$ is $G$-equivariant) which therefore descends to the quotient. In other words, there exists a symplectomorphism

$$
\left[f_{\mu}\right]: J_{1}^{-1}(\mu) / G_{\mu} \rightarrow J_{2}^{-1}(\mu) / G_{\mu}
$$


which is defined by $\left[f_{\mu}\right]\left(p_{M_{1}}(x)\right)=p_{M_{2}}(f(x))$, for all $x \in J_{1}^{-1}(\mu)$, where $p_{M_{i}}: M_{i} \rightarrow M_{i} / G$ are the canonical projections for $i \in\{1,2\}$.

\section{REDUCED LAGRANGIAN SUBMANIFOLDS}

In this section, we will prove a result which will be important for the rest of the paper. We will show that in the presence of a $G$-Hamiltonian action on a symplectic manifold $(M, \omega)$, a Lagrangian submanifold of $M$ can be reduced to a submanifold on the symplectic reduced space and that, under certain conditions, it retains its Lagrangian character.

We first need the following preparatory lemma.

Lemma 3.1. Let $\phi: G \times M \rightarrow M$ be a (free and proper) action of a Lie group $G$ on a manifold $M$ and $S$ be a $G$-invariant embedded (respectively connected, closed) submanifold of $M$. Then the quotient manifold $S / G$ is a embedded (respectively connected, closed) submanifold of $M / G$.

Proof. The action restricts to a (free and proper) action $\phi_{S}: G \times S \rightarrow S$, and therefore, $S / G$ is a smooth manifold. We will denote by $p_{S}: S \rightarrow S / G$ the canonical projection, by $i: S \rightarrow M$ the canonical inclusion of $S$ on $M$ and by $\widetilde{i}: S / G \rightarrow M / G$ the corresponding inclusion between the quotient manifolds.

Since $p_{S}: S \rightarrow S / G$ is a surjective submersion there exists, for all $x \in S$, an open subset $\widetilde{U} \subseteq S / G$ with $p_{S}(x) \in \widetilde{U}$ and a smooth local section $\widetilde{s}: \widetilde{U} \rightarrow S$ of $p_{S}$ satisfying $\widetilde{s}\left(p_{S}(x)\right)=x$. In fact,

$$
\widetilde{i}_{\mid \widetilde{U}}=p_{M} \circ i \circ \widetilde{s},
$$

where $p_{M}: M \rightarrow M / G$ is the canonical projection. This implies that the map $\widetilde{i}$ is smooth. Due to the fact that $i: S \rightarrow M$ is an immersion and due to the commutativity of the following diagram

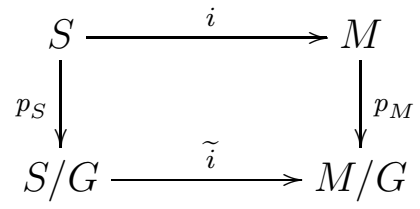

we obtain that $\widetilde{i}$ is an immersion as well. Next we will show that if $i: S \rightarrow M$ is an embedding, then $\tilde{i}: S / G \rightarrow M / G$ is also an embedding. Recall that the topology on $S / G$ is the final topology for the projection $p_{S}: S \rightarrow S / G$. This means that a set $\widetilde{U} \subseteq S / G$ is open in $S / G$ if, and only if, $p_{S}^{-1}(\widetilde{U})$ is open on $S$. Since $S$ has the induced topology by $M$, there exists an open subset $V$ on $M$ such that $p_{S}^{-1}(\widetilde{U})=V \cap S$. We also observe that $p_{M}: M \rightarrow M / G$ is an open map and thus $p_{M}(V)=\widetilde{V}$ is an open set of the quotient manifold $M / G$. Now, using that $S$ is $G$-invariant we conclude that $p_{M}(V \cap S)=\widetilde{V} \cap S / G$. This last statement follows from the relation $p_{M}(V \cap S)=p_{M}(G \cdot V \cap S)$. Therefore, $\widetilde{i}(\widetilde{U})=\widetilde{V} \cap S / G$. This concludes the proof that $\widetilde{i}$ is an embedding.

The statements about closedness and connectedness can readily be checked.

We are now ready to prove the main result we had announced at the beginning of the section. 
Theorem 3.2. Let $\phi: G \times M \rightarrow M$ be a (free and proper) G-Hamiltonian action on a symplectic manifold $(M, \omega)$ and let $J: M \rightarrow \mathfrak{g}^{*}$ be the corresponding Ad*-equivariant momentum map. Suppose that $S$ is a Lagrangian submanifold of $M$ which is closed, connected and embedded. Then:

(1) There exists a value $\mu \in \mathfrak{g}^{*}$ such that the submanifold $S$ is contained in the level set $J^{-1}(\mu)$.

(2) The space of orbits $S_{\mu}=S / G_{\mu}$ is an isotropic submanifold of the reduced symplectic manifold $\left(J^{-1}(\mu) / G_{\mu}, \omega_{\mu}\right)$.

(3) The submanifold $S_{\mu}$ is Lagrangian if, and only if, $\mathfrak{g}=\mathfrak{g}_{\mu}$.

Proof. Recall that $S$ being Lagrangian is equivalent to the following two conditions: $\operatorname{dim} S=1 / 2 \operatorname{dim} M$, and $S$ is isotropic, i.e.

$$
T_{x} S \subset\left(T_{x} S\right)^{\perp}=\left\{u \in T_{x} M: \omega(x)(u, v)=0, \quad \text { for all } v \in T_{x} S\right\},
$$

for all $x \in S$.

(1) We must prove that $J_{\mid S}: S \rightarrow \mathfrak{g}^{*}$ is a constant map or equivalently, that for each $\xi \in \mathfrak{g}$, the real function $J_{\xi \mid S}: S \rightarrow \mathbb{R}$ given by

$$
J_{\xi \mid S}(x)=\langle J(x), \xi\rangle \quad \text { for all } x \in S,
$$

is constant. Since $S$ is connected it suffices to show $d\left(J_{\xi}\right)_{\mid S}=0$. From the $G$ invariance of $S$, we have $\xi_{M}(x) \in T_{x} S$, and this fact, together with the isotropy condition on $S$ (namely $T_{x} S \subset\left(T_{x} S\right)^{\perp}$ ), implies

$\left\langle d\left(J_{\xi}\right)_{\mid S}(x), v\right\rangle=\left\langle\left(d J_{\xi}\right)(x), v\right\rangle=\left\langle\left(i_{\xi_{M}} \omega\right)(x), v\right\rangle=\omega\left(\xi_{M}(x), v\right)=0, \quad$ for $v \in T_{x} S$.

We conclude that there exists a $\mu \in \mathfrak{g}^{*}$ such that $S \subseteq J^{-1}(\mu)$.

(2) When we apply Lemma 3.1 to the induced $G_{\mu}$-action on $J^{-1}(\mu)$, it follows that $S_{\mu}=S / G_{\mu}$ is a closed connected embedded submanifold of the reduced symplectic manifold $\left(M_{\mu}=J^{-1}(\mu) / G_{\mu}, \omega_{\mu}\right)$.

Now, we will show that $S / G_{\mu}$ is an isotropic submanifold of $\left(J^{-1}(\mu) / G_{\mu}, \omega_{\mu}\right)$. This means that

$$
T_{\left(p_{S}(x)\right)} S / G_{\mu} \subseteq\left(T_{\left(p_{S}(x)\right)} S / G_{\mu}\right)^{\perp}, \quad \text { for all } p_{S}(x) \in S / G_{\mu},
$$

where $p_{S}: S \rightarrow S / G_{\mu}$ is the canonical projection and the orthogonality $\perp$ is understood with respect to the symplectic structure $\omega_{\mu}$. Let $u, v \in T_{x} S$, then $T_{x} p_{S}(u)$ and $T_{x} p_{S}(v)$ are elements of $T_{p_{S}(x)}(S / G)$. By considering the following commutative diagram

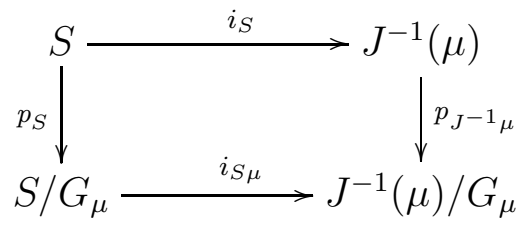

it follows that

$$
\begin{aligned}
\omega_{\mu}\left(p_{S}(x)\right)\left(T_{x} p_{S}(u), T_{x} p_{S}(v)\right) & =\omega_{\mu}\left(p_{J^{-1} \mu}(x)\right)\left(T_{x} p_{J^{-1} \mu}(u), T_{x} p_{J^{-1} \mu}(v)\right) \\
& =\left(\left(p_{J^{-1} \mu}\right)^{*} \omega_{\mu}\right)(x)(u, v) .
\end{aligned}
$$


Recall that the symplectic form $\omega_{\mu}$ on $J^{-1}(\mu) / G_{\mu}$ is characterized by $\left(p_{J^{-1} \mu}\right)^{*} \omega_{\mu}=$ $i^{*} \omega$, where $i: J^{-1}(\mu) \rightarrow M$ is the canonical inclusion. Then

$$
\left(\left(p_{J^{-1} \mu}\right)^{*} \omega_{\mu}\right)(x)(u, v)=\left(i^{*} \omega\right)(x)(u, v)=\omega(x)(u, v)=0,
$$

where in the last equality we have used the assumption that $S$ is Lagrangian (in particular, that it is isotropic).

(3) Since by assumption $\operatorname{dim} S=1 / 2 \operatorname{dim} M$ and since

$$
\begin{aligned}
\operatorname{dim}\left(J^{-1}(\mu) / G_{\mu}\right) & =\operatorname{dim} M-\operatorname{dim} G-\operatorname{dim} G_{\mu}, \\
\operatorname{dim}\left(S / G_{\mu}\right) & =\operatorname{dim} S-\operatorname{dim} G_{\mu},
\end{aligned}
$$

it follows that $S / G_{\mu}$ is Lagrangian if, and only if, $\operatorname{dim} G=\operatorname{dim} G_{\mu}$. In other words, $S / G_{\mu}$ is Lagrangian if, and only if, $\mathfrak{g}=\mathfrak{g}_{\mu}$.

Example 3.3. Let $\phi: G \times M \rightarrow M$ be an action of a Lie group $G$ on a connected manifold $M$ and $H \in C^{\infty}(M)$ a $G$-invariant function. Then, the image of the differential of $H, d H(M)$, is a Lagrangian submanifold of the cotangent bundle $\left(T^{*} M, \omega_{M}\right)$ which is invariant with respect to the cotangent lifted action $\phi^{T^{*} M}$ of $\phi$. Indeed,

$$
\phi_{g}^{T^{*} M}(d H(q))=d\left(H \circ \phi_{g^{-1}}\right)(g q)=d H(g q),
$$

for each $g \in G$ and $q \in M$, where the last equality holds by the invariance of the function $H$.

Applying Theorem 3.2 to the (closed, connected and embedded) Lagrangian submanifold $d H(M)$, there exists a value $\mu$ of the momentum map $J_{T^{*} M}: T^{*} M \rightarrow \mathfrak{g}$ such that $d H(M) \subseteq J_{T^{*} M}^{-1}(\mu)$. In fact,

$$
\left\langle J_{T^{*} M}(d H(q)), \xi\right\rangle=\left\langle d H(q), \xi_{M}(q)\right\rangle=\xi_{M}(H)(q)=0,
$$

for all $\xi \in \mathfrak{g}$ and $q \in M$, where again the last equality is a consequence of the invariance of $H$. This shows that $d H(M) \subseteq J_{T^{*} M}^{-1}(0)$, so in this particular case $\mu=0$.

Given that the submanifold $d H(M)$ is $G$-invariant, we may consider the reduced submanifold $d H(M) / G$ of the reduced symplectic manifold $J_{T^{*} M}^{-1}(0) / G$ and, in view of Theorem 3.2, $d H(M) / G$ is Lagrangian. Actually, as we have already seen (Example 2.2), $J_{T^{*} M}^{-1}(0) / G$ may be identified with $T^{*}(M / G)$ and, under this identification, the Lagrangian submanifold $d H(M) / G$ is just $d h(M / G)$, where $h: M / G \rightarrow \mathbb{R}$ is the reduced Hamiltonian induced by $H$.

\section{Hamilton-Poincaré Reduction}

In this section we will obtain an intrinsic description of the solutions of the HamiltonPoincaré equations.

Let $\phi: G \times M \rightarrow M$ be an action on the symplectic manifold $(M, \omega)$, and consider its tangent and cotangent lift to $T M$ and $T^{*} M$ respectively. Unlike the cotangent action, the tangent action is not always Hamiltonian. Only when $\phi$ is required to be Hamiltonian so will also be $\phi_{g}^{T M}=T \phi_{g}$, as we show next. 
We will make use the following result from [23]. Let $\omega$ be a closed two-form on a manifold $M$ and consider the vector bundle morphism

$$
b_{\omega}: T M \rightarrow T^{*} M
$$

induced by $\omega$, which sends $v_{x} \in T_{x} M$ to the 1-form defined by $\left\langle b_{\omega}\left(v_{x}\right), w_{x}\right\rangle=\omega\left(v_{x}, w_{x}\right)$, for all $w_{x} \in T_{x} M$. One can show that the canonical symplectic form $\omega_{M}$ of $T^{*} M$ and the complete lift $\omega^{c}$ of the closed two-form $\omega$ to $T M$ are related by the morphism $b_{\omega}$ in the following way:

$$
b_{\omega}^{*}\left(\omega_{M}\right)=-\omega^{c} .
$$

This equation may in fact be used as an alternative definition of the complete lift of the form $\omega$. From the definition of $b_{\omega}$, it is clear that it is a vector bundle isomorphism in case $\omega$ is non-degenerate. Combined with (4.1), this shows that $\omega^{c}$ is a symplectic form on $T M$ (and that $b_{\omega}$ is an anti-symplectomorphism).

Theorem 4.1. Let $(M, \omega)$ be a symplectic manifold with a Hamiltonian action $\phi: G \times$ $M \rightarrow M$ and equivariant momentum $J: M \rightarrow \mathfrak{g}^{*}$. Then:

(1) The vector bundle isomorphism $b_{\omega}: T M \rightarrow T^{*} M$ is $G$-equivariant with respect to the actions $\phi^{T M}: G \times T M \rightarrow T M$ and $\phi^{T^{*} M}: G \times T^{*} M \rightarrow T^{*} M$.

(2) $\phi^{T M}$ is a $G$-Hamiltonian action on the symplectic manifold $\left(T M, \omega^{c}\right)$ whose associated Ad $d^{*}$-equivariant momentum map $J_{T M}: T M \rightarrow \mathfrak{g}^{*}$ is given by

$$
\left\langle J_{T M}\left(v_{x}\right), \xi\right\rangle=v_{x}\left(J_{\xi}\right), \quad \text { for all } v_{x} \in T M \text { and for all } \xi \in \mathfrak{g} \text {. }
$$

Equivalently, $J_{T M}$ satisfies $J_{T M}=-J_{T^{*} M} \circ b_{\omega}$, where $J_{T^{*} M}: T^{*} M \rightarrow \mathfrak{g}^{*}$ is the momentum map associated with the symplectic action $\phi^{T^{*} M}$.

Proof. (1) If $x \in M$ and $g \in G$, using that the action $\phi: G \times M \rightarrow M$ is symplectic, it is straightforward that

$$
b_{\omega}\left(\phi_{g}(x)\right) \circ T_{x} \phi_{g}=T_{\phi_{g}(x)}^{*} \phi_{g^{-1}} \circ b_{\omega}(x) .
$$

(2) Recall that the equivariant momentum map associated to the cotangent action is given by (see Example 2.2)

$$
\left\langle J_{T^{*} M}\left(\alpha_{x}\right), \xi\right\rangle=\left\langle\alpha_{x}, \xi_{M}(x)\right\rangle, \quad \text { for all } \alpha_{x} \in T^{*} M \text { and for all } \xi \in \mathfrak{g} .
$$

Define $J_{T M}: T M \rightarrow \mathfrak{g}^{*}$ by the equality $J_{T^{*} M} \circ b_{\omega}=-J_{T M}$. Using that $b_{\omega}$ is an equivariant anti-symplectomorphism, it follows easily that $J_{T M}$ is an equivariant momentum map which satisfies

$$
\left\langle J_{T M}\left(v_{x}\right), \xi\right\rangle=-\left\langle b_{\omega}\left(v_{x}\right), \xi_{M}(x)\right\rangle=-\omega(x)\left(v_{x}, \xi_{M}(x)\right)=v_{x}\left(J_{\xi}\right),
$$

for all $v_{x} \in T M$ and $\xi \in \mathfrak{g}$.

Applying the previous theorem to the case of a cotangent bundle $\left(T^{*} Q, \omega_{Q}, J_{T^{*} Q}\right)$, it follows that the vector bundle isomorphism (which is an anti-symplectomorphism) $b_{\omega_{Q}}: T T^{*} Q$ $\rightarrow T^{*} T^{*} Q$ is $G$-equivariant with respect to the $G$-Hamiltonian actions $\phi^{T T^{*} Q}$ and $\phi^{T^{*} T^{*} Q}$ defined as the tangent and cotangent lift of $\phi^{T^{*} Q}$. Moreover $b_{\omega_{Q}}$ preserves the momentum maps of these actions in the way explained above, namely

$$
J_{T^{*} T^{*} Q} \circ b_{\omega_{Q}}=-J_{T T^{*} Q} .
$$


Here $J_{T^{*} T^{*} Q}$ and $J_{T T^{*} Q}$ are defined as

$$
\begin{aligned}
\left\langle J_{T^{*} T^{*} Q}\left(\beta_{\alpha_{q}}\right), \xi\right\rangle & =\left\langle\beta_{\alpha_{q}}, \xi_{T^{*} Q}\left(\alpha_{q}\right)\right\rangle, \\
\left\langle J_{T T^{*} Q}\left(v_{\alpha_{q}}\right), \xi\right\rangle & =v_{\alpha_{q}}\left(\left(J_{T^{*} Q}\right)_{\xi}\right),
\end{aligned}
$$

for all $\beta_{\alpha_{q}} \in T^{*} T^{*} Q, v_{\alpha_{q}} \in T T^{*} Q$ and $\xi \in \mathfrak{g}$. Using the results on symplectic reduction from Section 2, the symplectic orbit spaces $J_{T T^{*} Q}^{-1}(0) / G$ and $J_{T^{*} T^{*} Q}^{-1}(0) / G$ are anti-symplectomorphic via the map $\left[\left(b_{\omega_{Q}}\right)_{0}\right]: J_{T T^{*} Q}^{-1}(0) / G \rightarrow J_{T^{*} T^{*} Q}^{-1}(0) / G$ which is characterized by the condition

$$
\left[\left(b_{\omega_{Q}}\right)_{0}\right] \circ p_{J_{T T^{*} Q}^{-1}(0)}=p_{J_{T^{*} T^{*} Q}^{-1}(0)} \circ b_{\omega_{Q \mid J_{T T^{*} Q}^{-1}}(0)} \cdot
$$

Let us focus on the range of the map $\left[\left(b_{\omega_{Q}}\right)_{0}\right]$, i.e. the symplectic space $\left(J_{T^{*} T^{*} Q}^{-1}(0) / G\right.$, $\left.\left(\omega_{T^{*} Q}\right)_{0}\right)$ obtained by a cotangent reduction at the (regular) value $\mu=0$. In Example 2.2 we explained how this space is symplectomorphic to the canonical symplectic space $T^{*}\left(T^{*} Q / G\right)$, where the symplectomorphism $\Psi_{0}:\left(J_{T^{*} T^{*} Q}^{-1}(0) / G,\left(\omega_{T^{*} Q}\right)_{0}\right) \rightarrow\left(T^{*}\left(T^{*} Q / G\right)\right.$, $\left.\omega_{T^{*} Q / G}\right)$ is defined by

$$
\left\langle\Psi_{0}\left(p_{T_{T^{*} T^{*}}^{-1}(0)}\left(\alpha_{\beta_{q}}\right)\right), T_{\beta_{q}} p_{T^{*} Q}\left(v_{\beta_{q}}\right)\right\rangle=\left\langle\alpha_{\beta_{q}}, v_{\beta_{q}}\right\rangle,
$$

for all $\beta_{q} \in T^{*} Q, \alpha_{\beta_{q}} \in J_{T^{*} T^{*} Q}^{-1}(0)$ and $v_{\beta_{q}} \in T T^{*} Q$. On the other hand, the symplectic space on the domain of $\left[\left(b_{\omega_{Q}}\right)_{0}\right], J_{T T^{*} Q}^{-1}(0) / G$, is not symplectomorphic to a tangent bundle. However, it is possible to define a vector bundle morphism $\Xi$ (over the identity of $\left.T^{*} Q / G\right)$

$$
\Xi: J_{T T^{*} Q}^{-1}(0) / G \rightarrow T\left(T^{*} Q / G\right)
$$

which is characterized by the condition

$$
\Xi\left(p_{J_{T T^{*} Q}^{-1}(0)}\left(v_{\alpha_{q}}\right)\right)=T p_{T^{*} Q}\left(v_{\alpha_{q}}\right),
$$

for all $v_{\alpha_{q}} \in J_{T T^{*} Q}^{-1}(0)$.

Recall from Remark 2.1 that the orbit space $T^{*} Q / G$ can be endowed with a Poisson structure by imposing the projection $p_{T^{*} Q}: T^{*} Q \rightarrow T^{*} Q / G$ to be a Poisson epimorphism (see also [19]). Indeed, if $\{., .\}_{T^{*} Q}$ and $\{., .\}_{T^{*} Q / G}$ denote the Poisson brackets on $T^{*} Q$ and $T^{*} Q / G$ respectively, then

$$
\left\{\widehat{\varphi} \circ p_{T^{*} Q}, \widehat{\gamma} \circ p_{T^{*} Q}\right\}_{T^{*} Q}=\{\widehat{\varphi}, \widehat{\gamma}\}_{T^{*} Q / G} \circ p_{T^{*} Q}
$$

for all $\widehat{\varphi}, \widehat{\gamma} \in C^{\infty}\left(T^{*} Q / G\right)$. We will write $\sharp_{T^{*} Q / G}: T^{*}\left(T^{*} Q / G\right) \rightarrow T\left(T^{*} Q / G\right)$ for the vector bundle morphism induced by the Poisson structure on $T^{*} Q / G$ :

$$
\sharp_{T^{*} Q / G}(d \widehat{\varphi})=X_{\widehat{\varphi}}, \quad \text { for all } \widehat{\varphi} \in C^{\infty}\left(T^{*} Q / G\right),
$$

where $X_{\widehat{\varphi}}$ is the Hamiltonian vector field on $T^{*} Q / G$ given by

$$
X_{\widehat{\varphi}}(\widehat{\gamma})=\{\widehat{\gamma}, \widehat{\varphi}\}_{T^{*} Q / G}, \quad \text { for all } \widehat{\gamma} \in C^{\infty}\left(T^{*} Q / G\right) .
$$

Thus, if $X_{\left(\widehat{\varphi} \circ p_{T^{*} Q}\right)}$ is the Hamiltonian vector field on $T^{*} Q$ corresponding to the function $\widehat{\varphi} \circ p_{T^{*} Q} \in C^{\infty}\left(T^{*} Q\right)$, it follows that

$$
T p_{T^{*} Q} \circ X_{\left(\widehat{\varphi} \circ p_{T^{*} Q}\right)}=X_{\widehat{\varphi}} \circ p_{T^{*} Q} .
$$

The next lemma summarizes the relation between the maps introduced before. 
Lemma 4.2. The following diagram

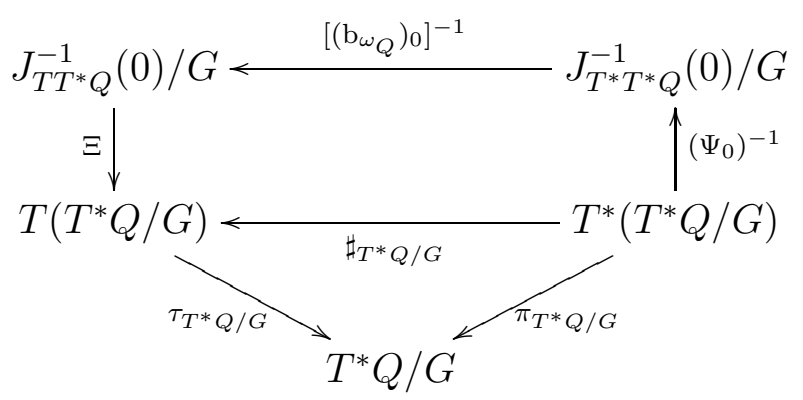

is commutative.

Proof. It is sufficient to prove that

$$
\sharp_{T^{*} Q / G}\left((d \widehat{\varphi})\left(p_{T^{*} Q}\left(\alpha_{q}\right)\right)\right)=\left(\Xi \circ\left[\left(b_{\omega_{Q}}\right)_{0}\right]^{-1} \circ \Psi_{0}^{-1}\right)\left((d \widehat{\varphi})\left(p_{T^{*} Q}\left(\alpha_{q}\right)\right)\right),
$$

for all $\widehat{\varphi} \in C^{\infty}\left(T^{*} Q / G\right)$ and $\alpha_{q} \in T^{*} Q$.

Consider the function $\widehat{\varphi} \circ p_{T^{*} Q} \in C^{\infty}\left(T^{*} Q\right)$. Then, it is clear that $d\left(\widehat{\varphi} \circ p_{T^{*} Q}\right)\left(\alpha_{q}\right) \in$ $J_{T^{*} T^{*} Q}^{-1}(0)$. Moreover, from the definition of $\Psi_{0}$ in (4.3) we have that for all $v_{\alpha_{q}} \in T T^{*} Q$

$$
\begin{aligned}
\left\langle\Psi_{0}\left(p_{J_{T^{*} T^{*} Q}^{-1}(0)}\left(d\left(\widehat{\varphi} \circ p_{T^{*} Q}\right)\left(\alpha_{q}\right)\right)\right), T_{\alpha_{q}} p_{T^{*} Q}\left(v_{\alpha_{q}}\right)\right\rangle & =\left\langle d\left(\widehat{\varphi} \circ p_{T^{*} Q}\right)\left(\alpha_{q}\right), v_{\alpha_{q}}\right\rangle \\
& =\left\langle d \widehat{\varphi}\left(p_{T^{*} Q}\left(\alpha_{q}\right)\right), T_{\alpha_{q}} p_{T^{*} Q}\left(v_{\alpha_{q}}\right)\right\rangle,
\end{aligned}
$$

and this means

$$
\Psi_{0}^{-1}\left((d \widehat{\varphi})\left(p_{T^{*} Q}\left(\alpha_{q}\right)\right)\right)=p_{J_{T^{*} T^{*} Q}^{-1}(0)}\left(d\left(\widehat{\varphi} \circ p_{T^{*} Q}\right)\left(\alpha_{q}\right)\right) .
$$

Combining the previous expression with the definition of $\left[\left(b_{\omega_{Q}}\right)_{0}\right]$ in (4.2) it follows that

$$
\left[\left(b_{\omega_{Q}}\right)_{0}\right]^{-1}\left(\Psi_{0}^{-1}\left((d \widehat{\varphi})\left(p_{T^{*} Q}\left(\alpha_{q}\right)\right)\right)\right)=p_{J_{T T^{*} Q}^{-1}(0)}\left(X_{\left(\widehat{\varphi} \circ p_{T^{*} Q}\right)}\left(\alpha_{q}\right)\right)
$$

and, recalling (4.4), we get:

$$
\left(\Xi \circ\left[\left(b_{\omega_{Q}}\right)_{0}\right]^{-1} \circ \Psi_{0}^{-1}\right)\left((d \widehat{\varphi})\left(p_{T^{*} Q}\left(\alpha_{q}\right)\right)\right)=\left(T_{\alpha_{q}} p_{T^{*} Q}\right)\left(X_{\left(\widehat{\varphi} \circ p_{T^{*} Q}\right)}\left(\alpha_{q}\right)\right) .
$$

Finally, taking into account (4.6), we conclude that

$$
\left(\Xi \circ\left[\left(b_{\omega_{Q}}\right)_{0}\right]^{-1} \circ \Psi_{0}^{-1}\right)\left((d \widehat{\varphi})\left(p_{T^{*} Q}\left(\alpha_{q}\right)\right)\right)=X_{\widehat{\varphi}}\left(p_{T^{*} Q}\left(\alpha_{q}\right)\right)=\left(\sharp_{T^{*} Q / G}\right)\left((d \widehat{\varphi})\left(p_{T^{*} Q}\left(\alpha_{q}\right)\right)\right) .
$$

Let $H: T^{*} Q \rightarrow \mathbb{R}$ be a $G$-invariant Hamiltonian and consider the $G$-invariant Lagrangian submanifold $d H\left(T^{*} Q\right) \subseteq J_{T^{*} T^{*} Q}^{-1}(0)$ (Example 3.3). The reduced submanifold $d H\left(T^{*} Q\right) / G$ is a Lagrangian submanifold of the reduced symplectic manifold $J_{T^{*} T^{*} Q}^{-1}(0) / G$ which can be mapped into a Lagrangian submanifold of $J_{T T^{*} Q}^{-1}(0) / G$ using the map $\left[\left(b_{\omega_{Q}}\right)_{0}\right]$. In other words, if we denote by $S_{H}$ the Lagrangian submanifold $\left(b_{\omega_{Q}}\right)^{-1}\left(d H\left(T^{*} Q\right)\right)$ of $\left(T T^{*} Q, \omega_{Q}^{c}\right)$, then

$$
S_{H} / G=\left[\left(b_{\omega_{Q}}\right)_{0}\right]^{-1}\left(d H\left(T^{*} Q\right) / G\right) \subset J_{T^{*} T^{*} Q}^{-1}(0) / G
$$

is again a Lagrangian submanifold which coincides with the submanifold

$$
S_{h}=\left(\left[\left(b_{\omega_{Q}}\right)_{0}\right]^{-1} \circ \Psi_{0}^{-1} \circ d h\right)\left(T^{*} Q / G\right) \subset J_{T^{*} T^{*} Q}^{-1}(0) / G,
$$

where $h: T^{*} Q / G \rightarrow \mathbb{R}$ is the reduced Hamiltonian. 
The results above imply the existence of a one-to-one correspondence between curves in $T^{*} Q / G$ and curves in the Lagrangian submanifold $S_{h}$. This correspondence is defined as follows: if $\gamma(t)$ is a curve in $T^{*} Q / G$, then

$$
t \rightarrow\left(\left[\left(b_{\omega_{Q}}\right)_{0}\right]^{-1} \circ \Psi_{0}^{-1} \circ d h\right)(\gamma(t))
$$

is the corresponding curve in $S_{h}$. Conversely, a curve $\bar{\gamma}(t)$ in $S_{h}$ projects onto a curve $\left(\pi_{T^{*} Q / G} \Psi_{0} \circ\left(b_{\omega_{Q}}\right)_{0}\right)(\bar{\gamma}(t))=\left(\tau_{T^{*} Q / G} \circ \Xi\right)(\bar{\gamma}(t))$ on $T^{*} Q / G$.

The next theorem relates this observation with the Hamilton-Poincaré equations. Roughly speaking, these equations follow from the symmetry reduction of Hamilton's equations. A geometric framework for these equation was first introduced in [5] and since then several different approaches have appeared in the literature. Here we shall use the following characterization from [14]: a curve $\gamma: I \rightarrow T^{*} Q / G$ is a solution of the Hamilton-Poincaré equations for $H$ if, and only if, $\gamma: I \rightarrow T^{*} Q / G$ is an integral curve of the Hamiltonian vector field $X_{h} \in \mathcal{X}\left(T^{*} Q / G\right)$ with respect to the linear Poisson structure on $T^{*} Q / G$, i.e.

$$
\sharp_{T^{*} Q / G}(d h(\gamma(t)))=X_{h}(\gamma(t))=\frac{d}{d t} \gamma(t) .
$$

Theorem 4.3. Let $H: T^{*} Q \rightarrow \mathbb{R}$ be a $G$-invariant Hamiltonian. Then, in the one-to-one correspondence between curves in $T^{*} Q / G$ and curves in $S_{h}$, the solutions of the HamiltonPoincaré equations correspond with curves in $S_{h}$ whose image by $\Xi$ are tangents lifts of curves in $T^{*} Q / G$.

Proof. If we consider a solution $\gamma: I \rightarrow T^{*} Q / G$ of the Hamilton-Poincaré equations, using (4.8) and Lemma 4.2 it follows that

$$
\left(\Xi \circ\left[\left(b_{\omega_{Q}}\right)_{0}\right]^{-1} \circ \Psi_{0}{ }^{-1}\right)(d h(\gamma(t)))=\sharp_{T^{*} Q / G}(d h(\gamma(t)))=\frac{d}{d t} \gamma(t) .
$$

Thus, if we take the curve $\bar{\gamma}: I \rightarrow S_{h}$ defined as

$$
\bar{\gamma}(t)=\left(\left[\left(b_{\omega_{Q}}\right)_{0}\right]^{-1} \circ \Psi_{0}^{-1}\right)(d h(\gamma(t)),
$$

we deduce that $\Xi \circ \bar{\gamma}$ is just the tangent lift of $\gamma$.

Conversely, let $\bar{\gamma}: I \rightarrow S_{h}$ be a curve on $S_{h}$ such that

$$
(\Xi \circ \bar{\gamma})(t)=\frac{d}{d t} \gamma(t)
$$

where $\gamma: I \rightarrow T^{*} Q / G$ is a curve on $T^{*} Q / G$. Then,

$$
\left(\tau_{T^{*} Q / G} \circ \Xi \circ \bar{\gamma}\right)(t)=\gamma(t)
$$

which implies that

$$
\bar{\gamma}(t)=\left(\left[\left(b_{\omega_{Q}}\right)_{0}\right]^{-1} \circ \Psi_{0}^{-1}\right)(d h(\gamma(t)) .
$$

As a consequence, $\gamma$ is the corresponding curve in $T^{*} Q / G$ associated with $\bar{\gamma}$ and

$$
\frac{d}{d t} \gamma(t)=(\Xi \circ \bar{\gamma})(t)=\sharp_{T^{*} Q / G}(d h(\gamma(t))) .
$$

We conclude that the curve $\gamma$ on $T^{*} Q / G$ solves the Hamilton-Poincaré equations for $H$.

Using the previous theorem, we obtain an intrinsic description of the Hamilton-Poincaré equations. 
Corollary 4.4. Let $H: T^{*} Q \rightarrow \mathbb{R}$ be a G-invariant Hamiltonian function. A curve $\gamma: I \rightarrow T^{*} Q / G$ is a solution of the Hamilton-Poincaré equations for $H$ if, and only if, the image of $\Xi$ by the corresponding curve in $S_{h}$,

$$
t \rightarrow \bar{\gamma}(t)=\left(\left[\left(b_{\omega_{Q}}\right)_{0}\right]^{-1} \circ \Psi_{0}^{-1} \circ d h\right)(\gamma(t)),
$$

is the tangent lift of $\gamma$.

The following diagram summarizes the results above:

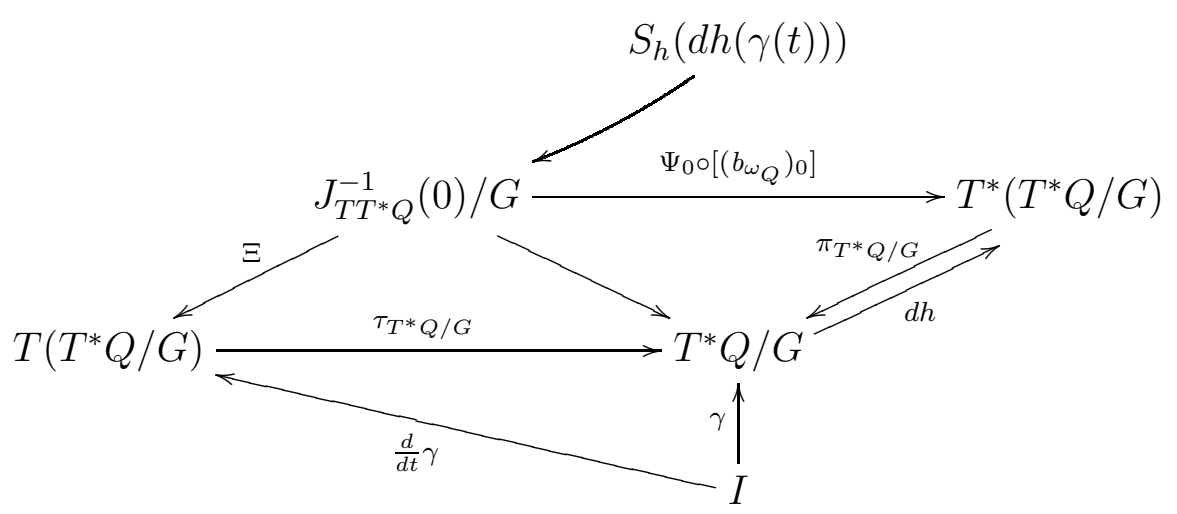

\section{Special Cases}

It is possible to give local expressions of the results above in full generality. This would lead to the coordinate version of the so-called vertical and horizontal Hamilton-Poincaré equations which can be found in e.g. [5, 14, 27, 21]. However in view of the many technicalities involved with these local computations (such as invoking a principal connection and its curvature, choosing adapted coordinates, etc.) we will only treat here two special cases.

5.1. The case where the configuration space is a Lie group. We will use the action by left translation on $G$. This will lead to an interpretation of the Lie-Poisson equations as distinguished curves in a Lagrangian submanifold. For the sake of clarity, we divide the example in 4 steps.

1) The vector Bundle isomorphism $b_{\omega_{G}}: T T^{*} G \rightarrow T^{*} T^{*} G$. It is well known that the cotangent bundle $T^{*} G$ of the Lie group $G$ may be identified with the trivial principal bundle with total space $G \times \mathfrak{g}^{*}$ and base $\mathfrak{g}^{*}$. Such identification is given by

$$
\alpha_{g} \in T_{g}^{*} G \rightarrow\left(g,\left(T_{e}^{*} L_{g}\right)\left(\alpha_{g}\right)\right) \in G \times \mathfrak{g}^{*} .
$$

In the same way, we will identify the tangent bundle $T G$ to $G$ with the trivial principal bundle with total space $G \times \mathfrak{g}$ and base space $\mathfrak{g}$. Combining these trivializations we further identify

$$
T T^{*} G \cong\left(G \times \mathfrak{g}^{*}\right) \times\left(\mathfrak{g} \times \mathfrak{g}^{*}\right), \quad T^{*} T^{*} G \cong\left(G \times \mathfrak{g}^{*}\right) \times\left(\mathfrak{g}^{*} \times \mathfrak{g}\right),
$$

whose elements will be denoted as follows:

$$
((g, \pi),(\omega, \dot{\pi})) \in\left(G \times \mathfrak{g}^{*}\right) \times\left(\mathfrak{g} \times \mathfrak{g}^{*}\right), \quad((g, \pi),(\tilde{\pi}, \omega)) \in\left(G \times \mathfrak{g}^{*}\right) \times\left(\mathfrak{g}^{*} \times \mathfrak{g}\right) .
$$

Under the identifications above it is obvious that the left translation on $G$ is mapped into the left translation onto the first factor, and that, therefore

$$
T^{*} G / G \cong \mathfrak{g}^{*}, \quad T T^{*} G / G \cong \mathfrak{g}^{*} \times\left(\mathfrak{g} \times \mathfrak{g}^{*}\right), \quad T^{*} T^{*} G / G \cong \mathfrak{g}^{*} \times\left(\mathfrak{g}^{*} \times \mathfrak{g}\right) .
$$


Using the definitions of the Liouville one-form and the canonical symplectic structure on $T^{*} G$, it follows that

$$
\begin{aligned}
\lambda_{G}(g, \pi)\left((g, \pi),\left(\omega_{1}, \dot{\pi}_{1}\right)\right) & =\left\langle\pi, \omega_{1}\right\rangle, \\
\omega_{G}(g, \pi)\left(\left((g, \pi),\left(\omega_{1}, \dot{\pi}_{1}\right)\right),\left((g, \pi),\left(\omega_{2}, \dot{\pi}_{2}\right)\right)\right) & =\left\langle\dot{\pi}_{2}, \omega_{1}\right\rangle-\left\langle\dot{\pi}_{1}, \omega_{2}\right\rangle+\left\langle\pi,\left[\omega_{1}, \omega_{2}\right]_{\mathfrak{g}}\right\rangle,
\end{aligned}
$$

for all $\left((g, \pi),\left(\omega_{1}, \dot{\pi}_{1}\right)\right),\left((g, \pi),\left(\omega_{2}, \dot{\pi}_{2}\right)\right) \in\left(G \times \mathfrak{g}^{*}\right) \times\left(\mathfrak{g} \times \mathfrak{g}^{*}\right) \cong T\left(T^{*} G\right)$.

Finally, from the expression of the canonical symplectic form $\omega_{G}$ it is straightforward that the vector bundle isomorphism

$$
b_{\omega_{G}}: T T^{*} G \cong\left(G \times \mathfrak{g}^{*}\right) \times\left(\mathfrak{g} \times \mathfrak{g}^{*}\right) \rightarrow T^{*} T^{*} G \cong\left(G \times \mathfrak{g}^{*}\right) \times\left(\mathfrak{g}^{*} \times \mathfrak{g}\right)
$$

is given by

$$
b_{\omega_{G}}((g, \pi),(\omega, \dot{\pi}))=\left((g, \pi),\left(-\dot{\pi}+a d_{\omega}^{*} \pi, \omega\right)\right),
$$

where $a d^{*}: \mathfrak{g} \times \mathfrak{g}^{*} \rightarrow \mathfrak{g}^{*}$ is the dual of the infinitesimal adjoint representation given by

$$
\left(a d_{\omega}^{*} \pi\right)(\tilde{\omega})=\left\langle\pi,[\omega, \tilde{\omega}]_{\mathfrak{g}}\right\rangle, \quad \text { for } \quad \omega, \tilde{\omega} \in \mathfrak{g} \quad \text { and } \pi \in \mathfrak{g}^{*} .
$$

2) The Reduced spaces $J_{T T^{*} G}^{-1}(0) / G$ AND $J_{T^{*} T^{*} G}^{-1}(0) / G$. Let $J_{T^{*} G}: T^{*} G \cong\left(G \times \mathfrak{g}^{*}\right) \rightarrow$ $\mathfrak{g}^{*}$ be the momentum map on $T^{*} G$, defined as

$$
\left\langle J_{T^{*} G}(g, \pi), \xi\right\rangle=\left\langle T_{g}^{*} L_{g^{-1}}(\pi), \xi_{G}(g)\right\rangle \quad \text { for all }(g, \pi) \in\left(G \times \mathfrak{g}^{*}\right) \text { and } \xi \in \mathfrak{g} .
$$

Since the action on $G$ is the left translation, its infinitesimal generators are the right invariant vector fields. Therefore

$$
\left\langle J_{T^{*} G}(g, \pi), \xi\right\rangle=\left\langle T_{g}^{*} L_{g^{-1}}(\pi), T_{e} R_{g}(\xi)\right\rangle=\left\langle\pi, A d_{g^{-1}} \xi\right\rangle=\left\langle A d_{g^{-1}}^{*} \pi, \xi\right\rangle,
$$

or, in other words, $J_{T^{*} G}(g, \pi)=A d_{g^{-1}}^{*} \pi$. With a similar computation we get the following expression for $J_{T^{*} T^{*} G}: T^{*} T^{*} G \cong\left(G \times \mathfrak{g}^{*}\right) \times\left(\mathfrak{g}^{*} \times \mathfrak{g}\right) \rightarrow \mathfrak{g}^{*}$ :

$$
J_{T^{*} T^{*} G}\left((g, \pi),\left(\pi^{\prime}, \omega\right)\right)=A d_{g^{-1}}^{*} \pi^{\prime} .
$$

In view of the expression for $b_{\omega_{G}}$ and Theorem 4.1, we immediately obtain the expression for the trivialized momentum $J_{T T^{*} G}$ :

$$
J_{T T^{*} G}((g, \pi),(\omega, \dot{\pi}))=A d_{g^{-1}}^{*}\left(\dot{\pi}-a d_{\omega}^{*} \pi\right) .
$$

In particular, on the zero level sets of the momenta, we have:

$$
\begin{aligned}
J_{T^{*} T^{*} G}^{-1}(0) & =\left\{((g, \pi),(0, \omega)) \in\left(G \times \mathfrak{g}^{*}\right) \times\left(\mathfrak{g}^{*} \times \mathfrak{g}\right)\right\} \cong\left(G \times \mathfrak{g}^{*}\right) \times \mathfrak{g}, \\
J_{T T^{*} G}^{-1}(0) & =\left\{\left((g, \pi),\left(\omega, a d_{\omega}^{*} \pi\right)\right) \in\left(G \times \mathfrak{g}^{*}\right) \times\left(\mathfrak{g} \times \mathfrak{g}^{*}\right)\right\} \cong G \times \mathfrak{g}^{*} \times \mathfrak{g},
\end{aligned}
$$

and therefore the reduced spaces

$$
\begin{aligned}
& J_{T^{*} T^{*} G}^{-1}(0) / G=\left\{\left(\pi,\left(\pi^{\prime}, \omega\right)\right) \in \mathfrak{g}^{*} \times\left(\mathfrak{g}^{*} \times \mathfrak{g}\right): \pi^{\prime}=0\right\} \cong \mathfrak{g}^{*} \times \mathfrak{g}, \\
& J_{T T^{*} G}^{-1}(0) / G=\left\{(\pi,(\omega, \dot{\pi})) \in \mathfrak{g}^{*} \times\left(\mathfrak{g} \times \mathfrak{g}^{*}\right): \dot{\pi}=a d_{\omega}^{*} \pi\right\} \cong \mathfrak{g}^{*} \times \mathfrak{g},
\end{aligned}
$$

can both be identified with $\mathfrak{g}^{*} \times \mathfrak{g}$.

3) The MAPs $\left[\left(b_{\omega_{G}}\right)_{0}\right], \Psi_{0}$ AND $\Xi$. In view of the above identifications and (5.1), the map $\left[\left(b_{\omega_{G}}\right)_{0}\right]$ is simply given by the identity

$$
\begin{aligned}
{\left[\left(b_{\omega_{G}}\right)_{0}\right]: \mathfrak{g}^{*} \times \mathfrak{g} } & \rightarrow \mathfrak{g}^{*} \times \mathfrak{g} \\
(\pi, \omega) & \mapsto\left[\left(b_{\omega_{G}}\right)_{0}\right](\pi, \omega)=(\pi, \omega) .
\end{aligned}
$$


On the other hand, we also have the identifications $T^{*}\left(T^{*} G / G\right) \cong \mathfrak{g}^{*} \times \mathfrak{g}$ and $T\left(T^{*} G / G\right) \cong$ $\mathfrak{g}^{*} \times \mathfrak{g}^{*}$, so we may as well work with trivialized expressions for the maps $\Psi_{0}$ and $\Xi$. One can check that these are given by:

$$
\begin{aligned}
\Psi_{0}: \mathfrak{g}^{*} \times \mathfrak{g} & \rightarrow \mathfrak{g}^{*} \times \mathfrak{g} \\
(\pi, \omega) & \mapsto \Psi_{0}(\pi, \omega)=(\pi, \omega), \\
\Xi: \mathfrak{g}^{*} \times \mathfrak{g} & \rightarrow \mathfrak{g}^{*} \times \mathfrak{g}^{*} \\
(\pi, \omega) & \mapsto \Xi(\pi, \omega)=\left(\pi, a d_{\omega}^{*} \pi\right) .
\end{aligned}
$$

4) The Lie-Poisson dynamics. Let $H: T^{*} G \cong G \times \mathfrak{g}^{*} \rightarrow \mathbb{R}$ be a $G$-invariant Hamiltonian and denote by $h: \mathfrak{g}^{*} \rightarrow \mathbb{R}$ the reduced Hamiltonian. Define the Lagrangian submanifold $S_{h}$ by (see Example 3.3):

$$
S_{h}=\left\{(\pi, d h(\pi)) \in \mathfrak{g}^{*} \times \mathfrak{g}\right\} \cong \mathfrak{g}^{*} .
$$

Consider a curve $\gamma(t)=(\pi(t), \omega(t)) \in J_{T T^{*} G}^{-1}(0) / G \cong \mathfrak{g}^{*} \times \mathfrak{g}$ with values in $S_{h}$ and which is such that its image by $\Xi$ is the tangent lift of a curve $t \mapsto \bar{\pi}(t) \in T^{*} G / G \cong \mathfrak{g}^{*}$. Then, it is clear that

$$
\pi(t)=\bar{\pi}(t), \quad \omega(t)=d h(\pi(t)), \quad a d_{\omega(t)}^{*} \pi(t)=\frac{d}{d t} \bar{\pi}(t) .
$$

Thus, it follows that

$$
a d_{d h(\pi(t))}^{*} \pi(t)=\frac{d}{d t} \pi(t) .
$$

Therefore, the curve $t \mapsto \pi(t)$ in $\mathfrak{g}^{*}$ solves the well known Lie-Poisson equations.

Conversely, assume that a curve in $\mathfrak{g}^{*}, t \mapsto \pi(t)$, is a solution of the Lie-Poisson equations for $H$ and consider the following curve in $S_{h}$ :

$$
\left.t \mapsto \gamma(t)=\left[\left(b_{\omega_{G}}\right)_{0}\right]^{-1} \circ \Psi_{0}^{-1}\right)\left(d h(\pi(t))=\left(\pi(t), d h(\pi(t)) \in \mathfrak{g}^{*} \times \mathfrak{g} \cong J_{T T^{*} G}^{-1}(0) / G .\right.\right.
$$

Its image by the map $\Xi$ is the curve

$$
t \mapsto\left(\pi(t), a d_{d h(\pi(t))}^{*} \pi(t)\right) \in \mathfrak{g}^{*} \times \mathfrak{g}^{*} \cong T\left(T^{*} G / G\right) .
$$

Using that $t \rightarrow \pi(t)$ is a solution of the Lie-Poisson equations, it follows that

$$
\frac{d \pi}{d t}_{\mid t}=a d_{d h(\pi(t))}^{*} \pi(t)
$$

i.e., the curve $\Xi \circ \gamma$ is the tangent lift of the curve $t \rightarrow \pi(t) \in \mathfrak{g}^{*}$.

5.2. The case where the configuration space is a product. The second example of interest is the case where the configuration space $Q$ can be written as $G \times S$ and the action of $G$ on $G \times S$ is the left translation on the first factor. If we denote by $\pi_{1}: Q \rightarrow G$ and $\pi_{2}: Q \rightarrow S$ the projections, the canonical symplectic form $\omega_{Q}$ in $T^{*} Q$ can be decomposed as $\omega_{Q}=\pi_{1}^{*} \omega_{G}+\pi_{2}^{*} \omega_{S}$ (where $\omega_{S}$ and $\omega_{G}$ can be interpreted as the canonical forms on $T^{*} S$ and $T^{*} G$, respectively) and that, as a result, we have

$$
b_{\omega_{Q}}=\left(b_{\omega_{G}}, b_{\omega_{S}}\right): T T^{*} G \times T T^{*} S \simeq T\left(T^{*} G \times T^{*} S\right) \rightarrow T^{*} T^{*} G \times T^{*} T^{*} S \simeq T^{*}\left(T^{*} G \times T^{*} S\right),
$$
where $b_{\omega_{S}}$ reads like $b_{\omega_{Q}}$ in Section 2 , with $Q$ replaced by $S$.

The momentum maps can be computed as before, in the case of a Lie group. In particular,

$$
\left\langle J_{T^{*} Q}\left((g, \pi), \alpha_{x}\right), \omega\right\rangle=\left\langle J_{T^{*} G}(g, \pi), \omega\right\rangle=\left\langle\pi, A d_{g^{-1}} \omega\right\rangle,
$$


for all $\left((g, \pi), \alpha_{x}\right) \in\left(G \times \mathfrak{g}^{*}\right) \times T^{*} S$, with $x \in S$. As a consequence we obtain directly the expressions for $J_{T T^{*} Q}$ and $J_{T^{*} T^{*} Q}$ from those in the previous subsection. For example, we find:

$$
J_{T T^{*} Q}\left(((g, \pi),(\omega, \dot{\pi})), X_{p_{x}}\right)=J_{T T^{*} G}((g, \pi),(\omega, \dot{\pi}))=A d_{g^{-1}}^{*}\left(\dot{\pi}-a d_{\omega}^{*} \pi\right),
$$

for all $\left(((g, \pi),(\omega, \dot{\pi})), X_{p_{x}}\right) \in\left(\left(G \times \mathfrak{g}^{*}\right) \times\left(\mathfrak{g} \times \mathfrak{g}^{*}\right)\right) \times T T^{*} S$, with $p_{x} \in T^{*} S$, and similarly for $J_{T^{*} T^{*} Q}$. Therefore, on the zero level sets we have the identifications:

$$
\begin{gathered}
J_{T T^{*} Q}^{-1}(0) / G \cong J_{T T^{*} G}^{-1}(0) / G \times T T^{*} S \cong\left(\mathfrak{g}^{*} \times \mathfrak{g}\right) \times T T^{*} S, \\
J_{T^{*} T^{*} Q}^{-1}(0) / G \cong J_{T^{*} T^{*} G}^{-1}(0) / G \times T^{*} T^{*} S \cong\left(\mathfrak{g}^{*} \times \mathfrak{g}\right) \times T^{*} T^{*} S .
\end{gathered}
$$

By taking the previous expressions into account, we deduce that the reduced map $\left[\left(b_{\omega_{Q}}\right)_{0}\right]$ is of the form

$$
\left[\left(b_{\omega_{Q}}\right)_{0}\right]=\left(\left[b_{\omega_{G}}\right]_{0}, b_{\omega_{S}}\right): J_{T T^{*} G}^{-1}(0) / G \times T T^{*} S \rightarrow J_{T^{*} T^{*} G}^{-1}(0) / G \times T^{*} T^{*} S .
$$

The map $\Psi_{0}$ is the identity and the map $\Xi: J_{T T^{*} Q}^{-1}(0) / G \simeq\left(\mathfrak{g}^{*} \times \mathfrak{g}\right) \times T T^{*} S \rightarrow$ $T\left(T^{*} Q / G\right) \simeq\left(\mathfrak{g}^{*} \times \mathfrak{g}^{*}\right) \times T T^{*} S$ is given by

$$
\Xi\left((\pi, \omega), X_{p_{x}}\right)=\left(\left(\pi, a d_{\omega}^{*} \pi\right), X_{p_{x}}\right) .
$$

Now, suppose that $H: T^{*} Q \simeq G \times \mathfrak{g}^{*} \times T^{*} S \rightarrow \mathbb{R}$ is a $G$-invariant Hamiltonian function and $h: T^{*} Q / G \simeq \mathfrak{g}^{*} \times T^{*} S \rightarrow \mathbb{R}$ is the reduced Hamiltonian function. Then, the Lagrangian submanifold $S_{h}$ in $J_{T T^{*} Q}^{-1}(0) / G \simeq\left(\mathfrak{g}^{*} \times \mathfrak{g}\right) \times T T^{*} S$ is given by

$$
S_{h}=\left\{\left(\pi, d h_{\alpha_{x}}(\pi), X_{h_{\pi}}\left(\alpha_{x}\right)\right) \mid \pi \in \mathfrak{g}^{*}, \alpha_{x} \in T^{*} S\right\}
$$

where $h_{\alpha_{x}}: \mathfrak{g}^{*} \rightarrow \mathbb{R}$ (respectively, $h_{\pi}: T^{*} S \rightarrow \mathbb{R}$ ) is the real function on $\mathfrak{g}^{*}$ (respectively, $T^{*} S$ ) defined by

$$
h_{\alpha_{x}}\left(\pi^{\prime}\right)=h\left(\pi^{\prime}, \alpha_{x}\right), \quad \text { for } \pi^{\prime} \in \mathfrak{g}^{*}
$$

(respectively, $h_{\pi}\left(\alpha_{x^{\prime}}^{\prime}\right)=h\left(\pi, \alpha_{x^{\prime}}^{\prime}\right)$, for $\alpha_{x^{\prime}}^{\prime} \in T^{*} S$ ), and $X_{h_{\pi}}$ is the Hamiltonian vector field in $T^{*} S$ of $h_{\pi}$.

Thus, a curve $t \mapsto\left(\pi(t), x(t), p_{x}(t)\right)$ on $\mathfrak{g}^{*} \times T^{*} S \simeq T^{*} Q / G$ satisfies the conditions of Corollary 4.4 if and only if

$$
\dot{\pi}=a d_{\frac{\partial h}{\partial \pi} \pi}^{*} \pi, \quad \dot{x}=\frac{\partial h}{\partial p_{x}}, \quad \dot{p}_{x}=-\frac{\partial h}{\partial x},
$$

which are the Hamilton-Poincaré equations for $H$ in this case.

\section{LAGRANGe-PoinCARÉ REDUCTION}

To get an intrinsic description of the reduced Lagrangian equations of motion we will proceed in a similar way as we have done before for the Hamiltonian case.

The first thing to prove is that Tulczyjew's diffeomorphism $A_{Q}: T T^{*} Q \rightarrow T^{*} T Q$ is $G$ equivariant and preserves the momentum maps associated to the actions on $T T^{*} Q$ and $T^{*} T Q$. The map $A_{Q}$ may be defined in several ways. One possibility is to define $A_{Q}$ as the composition of two anti-symplectomorphisms, as we will explain in the next paragraphs. For more details, see [9].

A first element we need is the vector bundle projection $v^{*}: T^{*} T Q \rightarrow T^{*} Q$, characterized as

$$
\left\langle v^{*}\left(\alpha_{v_{q}}\right), w_{q}\right\rangle=\left\langle\alpha_{v_{q}},\left(w_{q}\right)_{v_{q}}^{\mathrm{v}}\right\rangle,
$$


for all $\alpha_{v_{q}} \in T^{*} T Q$ and $w_{q} \in T Q$, where $(\cdot)_{v_{q}}^{v}: T_{q} Q \rightarrow T_{v_{q}} T Q$ is the standard vertical lift:

$$
\left(w_{q}\right)_{v_{q}}^{v}(f)=\frac{d}{d s \mid s=0}\left(f\left(v_{q}+s w_{q}\right)\right),
$$

for each function $f$ on $T Q$.

The second element is a vector bundle isomorphism $R: T^{*} T Q \rightarrow T^{*} T^{*} Q$ over the identity of $T^{*} Q$ between the vector bundles $\mathrm{v}^{*}: T^{*} T Q \rightarrow T^{*} Q$ and $\pi_{T^{*} Q}: T^{*} T^{*} Q \rightarrow T^{*} Q$. It is completely determined by the condition:

$$
\left\langle R\left(\alpha_{v_{q}}\right), W_{\mathrm{v}^{*}\left(\alpha_{v_{q}}\right)}\right\rangle=-\left\langle\alpha_{v_{q}}, \bar{W}_{v_{q}}\right\rangle+\left\langle W_{\mathrm{v}^{*}\left(\alpha_{v_{q}}\right)}, \bar{W}_{v_{q}}\right\rangle^{T},
$$

for all $\alpha_{v_{q}} \in T^{*} T Q, \bar{W}_{v_{q}} \in T T Q$ and $W_{v^{*}\left(\alpha_{v_{q}}\right)} \in T T^{*} Q$ satisfiying

$$
T \tau_{Q}\left(\bar{W}_{v_{q}}\right)=T \pi_{Q}\left(W_{v^{*}\left(\alpha_{v_{q}}\right)}\right) .
$$

Here, $\langle\cdot, \cdot\rangle^{T}: T T^{*} Q \times_{T Q} T T Q \rightarrow \mathbb{R}$ is the pairing defined by the tangent map of the usual pairing $\langle\cdot, \cdot\rangle: T^{*} Q \times{ }_{Q} T Q \rightarrow \mathbb{R}$.

The Tulczyjew diffeomorphism $A_{Q}$ is then defined as the composition $R^{-1} \circ b_{\omega_{Q}}$.

Lemma 6.1. Consider the anti-symplectomorphism $R: T^{*} T Q \rightarrow T^{*} T^{*} Q$. Then:

(1) $R$ is G-equivariant.

(2) $R$ satisfies $J_{T^{*} T^{*} Q} \circ R=-J_{T^{*} T Q}$

Proof. (1) First, we check that the map v*: $T^{*} T Q \rightarrow T^{*} Q$ is $G$-equivariant. It can readily be checked that the vertical lift is $G$-equivariant, or in short $g\left(w_{q}\right)_{v_{q}}^{v}=\left(g w_{q}\right)_{g v_{q}}^{v}$. Thus, for all $\alpha_{v_{q}} \in T^{*} T Q, w_{q} \in T Q$ and $g \in G$,

$$
\begin{aligned}
\left\langle\mathrm{v}^{*}\left(g \alpha_{v_{q}}\right),\left(g w_{q}\right)\right\rangle & =\left\langle g \alpha_{v_{q}},\left(g w_{q}\right)_{g v_{q}}^{\mathrm{v}}\right\rangle=\left\langle g \alpha_{v_{q}}, g\left(w_{q}\right)_{v_{q}}^{\mathrm{v}}\right\rangle \\
& =\left\langle\alpha_{v_{q}},\left(w_{q}\right)_{v_{q}} v^{\mathrm{v}}\right\rangle=\left\langle\mathrm{v}^{*}\left(\alpha_{v_{q}}\right),\left(w_{q}\right)\right\rangle,
\end{aligned}
$$

where we have used invariance of the natural pairing.

Secondly we check equivariance of $R$. Using the equivariance of the maps $T \tau_{Q}$ and $T \pi_{Q}$ and the invariance of the pairings, we find

$$
\begin{aligned}
\left\langle R\left(g \alpha_{v_{q}}\right), g\left(W_{\mathrm{v}^{*}\left(\alpha_{v_{q}}\right)}\right)\right\rangle & =-\left\langle g \alpha_{v_{q}}, g \bar{W}_{v_{q}}\right\rangle+\left\langle g W_{\mathrm{v}^{*}\left(\alpha_{v_{q}}\right)}, g \bar{W}_{v_{q}}\right\rangle^{T} \\
& =-\left\langle\alpha_{v_{q}}, \bar{W}_{v_{q}}\right\rangle+\left\langle W_{\mathrm{v}^{*}\left(\alpha_{v_{q}}\right)}, \bar{W}_{v_{q}}\right\rangle^{T},
\end{aligned}
$$

from where $R\left(g \alpha_{v_{q}}\right)=g R\left(\alpha_{v_{q}}\right)$ follows.

(2) From the definition of the momentum map $J_{T^{*} T^{*} Q}$ it follows that for all $\alpha_{v_{q}} \in T^{*} T Q$ and $\xi \in \mathfrak{g}$,

$$
\left\langle J_{T^{*} T^{*} Q}\left(R\left(\alpha_{v_{q}}\right)\right), \xi\right\rangle=\left\langle R\left(\alpha_{v_{q}}\right), \xi_{T^{*} Q}\left(\mathrm{v}^{*}\left(\alpha_{v_{q}}\right)\right)\right\rangle .
$$

Recalling the definition 6.2 of $R$ for $W=\xi_{T^{*} Q}\left(\mathrm{v}^{*}\left(\alpha_{v_{q}}\right)\right)$ and $\bar{W}=\xi_{T Q}\left(v_{q}\right)$ (note that this choice satisfies ([6.3) $)$, it follows that:

$$
\left\langle R\left(\alpha_{v_{q}}\right), \xi_{T^{*} Q}\left(\mathrm{v}^{*}\left(\alpha_{v_{q}}\right)\right)\right\rangle=-\left\langle\alpha_{v_{q}}, \xi_{T Q}\left(v_{q}\right)\right\rangle+\left\langle\xi_{T^{*} Q}\left(\mathrm{v}^{*}\left(\alpha_{v_{q}}\right)\right), \xi_{T Q}\left(v_{q}\right)\right\rangle^{T} .
$$


We now show that the last term vanishes. If we write $\varphi_{t}$ for the flow of $\xi_{Q}$ around $q \in$ $Q$, i.e. $\varphi_{t}=\exp (t \xi) q$, then the flows of $\xi_{T Q}$ and $\xi_{T^{*} Q}$ are $T \varphi_{t}$ and $T^{*} \varphi_{t}$ respectively. With this, using the invariance of the bracket, we conclude:

$$
\begin{aligned}
\left\langle\xi_{T^{*} Q}\left(\mathrm{v}^{*}\left(\alpha_{v_{q}}\right)\right), \xi_{T Q}\left(v_{q}\right)\right\rangle^{T} & =\frac{d}{d t}{ }_{\mid t=0}\left\langle\left(T^{*} \varphi_{t}\right)\left(\mathrm{v}^{*}\left(\alpha_{v_{q}}\right)\right), T \varphi_{t}\left(v_{q}\right)\right\rangle \\
& =\frac{d}{d t}{ }_{t=0}\left\langle\mathrm{v}^{*}\left(\alpha_{v_{q}}\right), v_{q}\right\rangle=0 .
\end{aligned}
$$

Therefore,

$$
\left\langle J_{T^{*} T^{*} Q}\left(R\left(\alpha_{v_{q}}\right)\right), \xi\right\rangle=-\left\langle\alpha_{v_{q}}, \xi_{T Q}\left(v_{q}\right)\right\rangle=-\left\langle J_{T^{*} T Q}\left(\alpha_{v_{q}}\right), \xi\right\rangle .
$$

The previous lemma implies the following important result:

Theorem 6.2. Tulczyjew's diffeomorphism is G-equivariant with respect to the G-Hamiltonian actions on $T T^{*} Q$ and $T^{*} T Q$ and, moreover, $J_{T T^{*} Q}=J_{T^{*} T Q} \circ A_{Q}$.

Proof. This is now an easy consequence of Theorem 4.1 and Lemma 6.1.

The results above imply the existence of the reduced maps

$$
\begin{aligned}
{\left[R_{0}\right]: } & J_{T^{*} T Q}^{-1}(0) / G \rightarrow J_{T^{*} T^{*} Q}^{-1}(0) / G, \\
{\left[\left(A_{Q}\right)_{0}\right]: } & J_{T T^{*} Q}^{-1}(0) / G \rightarrow J_{T^{*} T Q}^{-1}(0) / G,
\end{aligned}
$$

defined by

$$
\left[R_{0}\right] \circ p_{J_{T^{*} T Q}^{-1}(0)}=p_{J_{T^{*} T^{*} Q}^{-1}(0)} \circ R_{\mid J_{T^{*} T Q}^{-1}(0)}
$$

and

$$
\left[\left(A_{Q}\right)_{0}\right] \circ p_{J_{T T^{*} Q}^{-1}(0)}=p_{J_{T^{*} T Q}^{-1}(0)} \circ A_{Q_{\mid J_{T T^{*}}^{-1}}(0)},
$$

respectively. Taking into account the definition of Tulczyjew's diffeomorphism, it is clear that

$$
\left[\left(A_{Q}\right)_{0}\right]=\left[R_{0}\right]^{-1} \circ\left[\left(b_{\omega_{Q}}\right)_{0}\right]
$$

In fact we could have taken the above expression as an alternative definition. It is then analogous to the definition of the Tulczyjew's diffeomorphism. For this reason, we shall refer to $\left[\left(A_{Q}\right)_{0}\right]$ as the reduced Tulczyjew diffeomorphism.

Before we enter the discussion about Lagrange-Poincaré reduction we need to introduce a few more maps. Let us consider the manifold $\left(J_{T^{*} T Q}^{-1}(0) / G,\left(\omega_{T Q}\right)_{0}\right)$ (which is obtained after a cotangent reduction at $\mu=0)$ and the symplectomorphism

$$
\varphi_{0}:\left(J_{T^{*} T Q}^{-1}(0) / G,\left(\omega_{T Q}\right)_{0}\right) \rightarrow\left(T^{*}(T Q / G), \omega_{T Q / G}\right)
$$

defined by (see Example 2.2):

$$
\left\langle\varphi_{0}\left(p_{T_{T^{*} T Q}^{-1}(0)}\left(\alpha_{v_{q}}\right)\right), T_{v_{q}} p_{T Q}\left(u_{v_{q}}\right)\right\rangle:=\left\langle\alpha_{v_{q}}, u_{v_{q}}\right\rangle,
$$

for all $\alpha_{v_{q}} \in J_{T^{*} T Q}^{-1}(0)$ and $u_{v_{q}} \in T T Q$.

Next there is a vector bundle morphism $\Lambda: T^{*}(T Q / G) \rightarrow T\left(T^{*} Q / G\right)$ (over the identity in $T^{*} Q / G$ ) defined by

$$
\Lambda=\sharp_{T^{*} Q / G} \circ R_{Q / G} \text {. }
$$


Here, $\sharp_{T^{*} Q / G}: T^{*}\left(T^{*} Q / G\right) \rightarrow T\left(T^{*} Q / G\right)$ is the vector bundle morphism induced by the Poisson structure on $T^{*} Q / G$ (see (4.5)) and the isomorphism

$$
R_{Q / G}: T^{*}(T Q / G) \rightarrow T^{*}\left(T^{*} Q / G\right)
$$

will be described next (see [11] for a general definition).

First of all, we describe how $T^{*}(T Q / G)$ can be interpreted as a vector bundle over $T^{*} Q / G$. Note that $T^{*}(T Q / G)$ is a vector subbundle (over $T Q / G$ ) of the vector bundle $\left[\pi_{T Q}\right]: T^{*} T Q / G \rightarrow T Q / G$, with inclusion

$$
i: T^{*}(T Q / G) \rightarrow T^{*} T Q / G
$$

defined by

$$
i\left(\alpha_{p_{T Q}\left(v_{q}\right)}\right)=p_{T^{*} T Q}\left(\left(T_{v_{q}}^{*} p_{T Q}\right)\left(\alpha_{p_{T Q}\left(v_{q}\right)}\right)\right),
$$

for $\alpha_{p_{T Q}\left(v_{q}\right)} \in T^{*}(T Q / G)$ and $v_{q} \in T Q$. $T^{*} T Q / G$ is a vector bundle over $T^{*} Q / G$ and with vector bundle projection $\left[\mathrm{v}^{*}\right]: T^{*} T Q / G \rightarrow T^{*} Q / G$ induced by $\mathrm{v}^{*}$. Let the vector bundle projection

$$
\widetilde{\mathrm{v}^{*}}: T^{*}(T Q / G) \rightarrow T^{*} Q / G
$$

be the composition

$$
\widetilde{\mathrm{v}^{*}}=\left[\mathrm{v}^{*}\right] \circ i .
$$

We can now mimic the construction of $R$ to introduce the vector bundle map $R_{Q / G}$. Explicitly, $R_{Q / G}: T^{*}(T Q / G) \rightarrow T^{*}\left(T^{*} Q / G\right)$ is the isomorphism between the vector bundles $\widetilde{v^{*}}: T^{*}(T Q / G) \rightarrow T^{*} Q / G$ and $\pi_{T^{*} Q / G}: T^{*}\left(T^{*} Q / G\right) \rightarrow T^{*} Q / G$ such that:

$$
\begin{aligned}
& \left\langle R_{Q / G}\left(\alpha_{p_{T Q}\left(v_{q}\right)}\right), W_{{\widetilde{v^{*}}}^{*}\left(\alpha_{p_{T Q}\left(v_{q}\right)}\right)}\right\rangle= \\
& \quad-\left\langle\alpha_{p_{T Q}\left(v_{q}\right)}, \bar{W}_{p_{T Q}\left(v_{q}\right)}\right\rangle+\left\langle W_{{\widetilde{v^{*}}}\left(\alpha_{p_{T Q}\left(v_{q}\right)}\right)}, \bar{W}_{p_{T Q}\left(v_{q}\right)}\right\rangle^{T},
\end{aligned}
$$

for all $\alpha_{p_{T Q}\left(v_{q}\right)} \in T^{*}(T Q / G)$ and $W_{\widetilde{v}^{*}\left(\alpha_{p_{T Q}\left(v_{q}\right)}\right)} \in T\left(T^{*} Q / G\right)$, with $\bar{W}_{p_{T Q}\left(v_{q}\right)} \in T(T Q / G)$ satisfying

$$
T\left[\tau_{Q}\right]\left(\bar{W}_{p_{T Q}\left(v_{q}\right)}\right)=T\left[\pi_{Q}\right]\left(W_{\widetilde{v}^{*}\left(\alpha_{p_{T Q}\left(v_{q}\right)}\right)}\right),
$$

where $\left[\tau_{Q}\right]: T Q / G \rightarrow Q / G$ and $\left[\pi_{Q}\right]: T^{*} Q / G \rightarrow Q / G$ are the canonical projections and $\langle\cdot, \cdot\rangle^{T}: T\left(T^{*} Q / G\right) \times_{T(Q / G)} T(T Q / G) \rightarrow \mathbb{R}$ is the tangent map of the natural pairing $\langle\cdot, \cdot\rangle: T^{*} Q / G \times_{Q / G} T Q / G \rightarrow \mathbb{R}$.

The relation between the different maps introduced so far is summarized in the following Lemma:

Lemma 6.3. The diagram

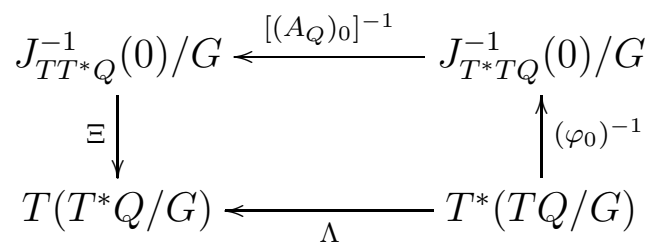

is commutative. 
Proof. On the one hand, we know that the inverse of the reduced Tulczyjew diffeomorphism satisfies $\left[\left(A_{Q}\right)_{0}\right]^{-1}=\left[\left(b_{\omega_{Q}}\right)_{0}\right]^{-1} \circ\left[R_{0}\right]$. On the other hand, we also know from Lemma 4.2 that the diagram

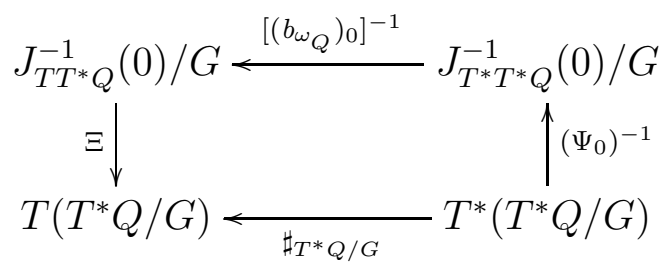

is commutative. Therefore, it is sufficient to check that the diagram

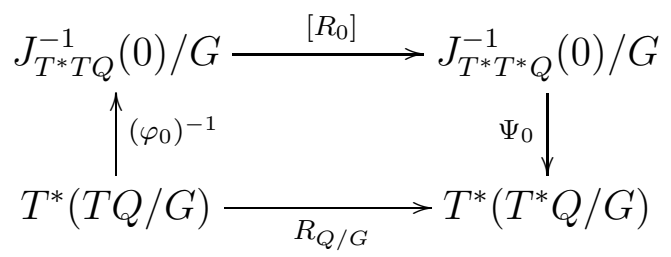

is commutative, for then the result will follow directly from diagram chasing:

$$
\Lambda=\sharp_{T^{*} Q / G} \circ R_{Q / G}=\left(\Xi \circ\left[\left(b_{\omega_{Q}}\right)_{0}\right]^{-1} \circ \Psi_{0}^{-1}\right) \circ\left(\Psi_{0} \circ\left[R_{0}\right] \circ \varphi_{0}^{-1}\right)=\Xi \circ\left[\left(A_{Q}\right)_{0}\right]^{-1} \circ \varphi_{0}^{-1} .
$$

Let $v_{q} \in T Q, W_{\mathrm{v}^{*}\left(\alpha_{v_{q}}\right)} \in T T^{*} Q$ and $f \in C^{\infty}(T Q / G)$ arbitrary. Using (4.3), (6.4) and (6.5), we have that

$$
\begin{aligned}
\left\langle\left(\Psi_{0} \circ\left[R_{0}\right] \circ \varphi_{0}^{-1}\right)\left(d f\left(p_{T Q}\left(v_{q}\right)\right)\right), T p_{T^{*} Q}\left(W_{\mathrm{v}^{*}\left(\alpha_{v_{q}}\right)}\right)\right\rangle= \\
=\left\langle\left(\Psi_{0} \circ\left[R_{0}\right]\right)\left(p_{J_{T^{*} T Q}^{-1}(0)}\left(d\left(f \circ p_{T Q}\right)\left(v_{q}\right)\right)\right), T p_{T^{*} Q}\left(W_{\mathrm{v}^{*}\left(\alpha_{v_{q}}\right)}\right)\right\rangle \\
=\left\langle\left(\Psi_{0} \circ p_{J_{T^{*} T^{*} Q}^{-1}(0)} \circ R\right)\left(d\left(f \circ p_{T Q}\right)\left(v_{q}\right)\right), T p_{T^{*} Q}\left(W_{\mathrm{v}^{*}\left(\alpha_{v_{q}}\right)}\right)\right\rangle \\
=\left\langle R\left(d\left(f \circ p_{T Q}\right)\left(v_{q}\right)\right), W_{\mathrm{v}^{*}\left(\alpha_{v_{q}}\right)}\right\rangle .
\end{aligned}
$$

Now, take $\bar{W}_{v_{q}} \in T T Q$ satisfying (6.3). From (6.2), it follows that

$$
\begin{aligned}
&\langle R(\left.\left.d\left(f \circ p_{T Q}\right)\left(v_{q}\right)\right), W_{\mathrm{v}^{*}\left(\alpha_{v_{q}}\right)}\right\rangle= \\
& \quad=-\left\langle d\left(f \circ p_{T Q}\right)\left(v_{q}\right), \bar{W}_{v_{q}}\right\rangle+\left\langle W_{\mathrm{v}^{*}\left(\alpha_{v_{q}}\right)}, \bar{W}_{v_{q}}\right\rangle^{T} \\
& \quad=-\left\langle d f\left(p_{T Q}\left(v_{q}\right)\right), T p_{T Q}\left(\bar{W}_{v_{q}}\right)\right\rangle+\left\langle T p_{T^{*} Q} W_{\mathrm{v}^{*}\left(\alpha_{v_{q}}\right)}, T p_{T Q}\left(\bar{W}_{v_{q}}\right)\right\rangle^{T} \\
& \quad=\left\langle R_{Q / G}\left(d f\left(p_{T Q}\left(v_{q}\right)\right)\right), T p_{T^{*} Q}\left(W_{\mathrm{v}^{*}\left(\alpha_{v_{q}}\right)}\right)\right\rangle .
\end{aligned}
$$

where in the equality between the second and the third lines, we have used that if $\langle\cdot, \cdot\rangle^{T}$ : $T(T Q) \times_{T Q} T\left(T^{*} Q\right) \rightarrow \mathbb{R}$ and $\langle\cdot, \cdot\rangle^{\hat{T}}: T(T Q / G) \times_{T(Q / G)} T\left(T^{*} Q / G\right) \rightarrow \mathbb{R}$ are the canonical pairings then

$$
\left\langle X_{v_{q}}, Y_{\alpha_{q}}\right\rangle^{T}=T\langle\cdot, \cdot\rangle\left(X_{v_{q}}, Y_{\alpha_{q}}\right)=\left\langle\left(T_{v_{q}} p_{T Q}\right)\left(X_{v_{q}}\right),\left(T_{\alpha_{q}} p_{T^{*} Q}\right)\left(Y_{\alpha_{q}}\right)\right\rangle^{\hat{T}},
$$

for $X_{v_{q}} \in T(T Q)$ and $Y_{\alpha_{q}} \in T\left(T^{*} Q\right)$ such that $\left(T_{v_{q}} p_{T Q}\right)\left(X_{v_{q}}\right)=\left(T_{\alpha_{q}} p_{T^{*} Q}\right)\left(Y_{\alpha_{q}}\right)$.

This concludes the proof. 
Let $L: T Q \rightarrow \mathbb{R}$ be a $G$-invariant Lagrangian function. Much like in the Hamiltonian case, we can show that $d L(T Q) / G$ is a Lagrangian submanifold of $J_{T^{*} T Q}^{-1}(0) / G$ which, by means of the reduced Tulczyjew's diffeomorphism, can be mapped into Lagrangian submanifold of $J_{T T^{*} Q}^{-1}(0) / G$. More precisely, let $S_{L}$ be the invariant Lagrangian submanifold of the symplectic manifold $\left(T T^{*} Q, \omega_{Q}^{c}\right)$ defined by $S_{L}=\left(A_{Q}\right)^{-1}(d L(T Q))$. The space of orbits $S_{L} / G$ is Lagrangian submanifold of $J_{T T^{*} Q}^{-1}(0) / G$ which satisfies:

$$
S_{L} / G=\left[\left(A_{Q}\right)_{0}\right]^{-1}(d L(T Q) / G) .
$$

An alternative description of this submanifold is:

$$
S_{L} / G=S_{l}:=\left(\left[\left(A_{Q}\right)_{0}\right]^{-1} \circ \varphi_{0}^{-1} \circ d l\right)(T Q / G),
$$

where $l: T Q / G \rightarrow \mathbb{R}$ is the reduced Lagrangian function characterized by the condition $L=l \circ p_{T Q}$.

This implies the existence of a one-to-one correspondence between curves in $T Q / G$ and curves in the Lagrangian submanifold $S_{l}$ defined as follows: if $\gamma: I \rightarrow T Q / G$ is a curve in $T Q / G$, then

$$
t \rightarrow\left(\left[\left(A_{Q}\right)_{0}\right]^{-1} \circ \varphi_{0}^{-1} \circ d l\right)(\gamma(t))
$$

is the corresponding curve in $S_{l}$.

As was the case for the Hamilton-Poincaré equations, there exist many different geometric frameworks for Lagrange-Poincaré reduction. We will use a somewhat indirect approach. In [14] it is shown that the Lagrange-Poincaré equations can be thought of as EulerLagrange equations on a Lie algebroid, where the Lie algebroid is the Atiyah algebroid $T Q / G$. In [9] the authors give a characterization of the set of solutions of the EulerLagrange equations on a Lie algebroid, which applied to the case of the Atiyah algebroid is as follows: a curve $\sigma: I \rightarrow T Q / G$ is a solution of the Lagrange-Poincaré equations if, and only if, it satisfies the equation

$$
\frac{d}{d t}\left(\mathcal{F}_{l} \circ \sigma\right)(t)=\Lambda(d l(\sigma(t))
$$

where $\mathcal{F}_{l}: T Q / G \rightarrow T^{*} Q / G$ is the Legendre transformation associated with $l$ defined by

$$
\mathcal{F}_{l}\left(p_{T Q}\left(v_{q}\right)\right)=\widetilde{\mathrm{v}^{*}}\left(d l\left(p_{T Q}\left(v_{q}\right)\right)\right),
$$

for all $v_{q} \in T Q$.

Theorem 6.4. Let $L: T Q \rightarrow \mathbb{R}$ be a G-invariant Lagrangian function. Then, in the one-to-one correspondence between curves in $T Q / G$ and curves in $S_{l}$, the solutions of the Lagrange-Poincaré equations correspond with curves in $S_{l}$ whose image by $\Xi$ are tangent lifts of curves in $T^{*} Q / G$.

Proof. Let us assume that a curve $\sigma: I \rightarrow T Q / G$ is a solution of the Lagrange-Poincaré equations for $L$. Then, using (6.9) and Lemma 6.3, it follows that

$$
\frac{d}{d t}\left(\mathcal{F}_{l}(\sigma(t))\right)=\Lambda(d l(\sigma(t)))=\left(\Xi \circ\left[\left(A_{Q}\right)_{0}\right]^{-1} \circ \varphi_{0}^{-1}\right)(d l(\sigma(t))) .
$$

Thus, if we take the curve $\bar{\sigma}: I \rightarrow S_{l}$ in $S_{l}$ associated with $\sigma$

$$
\bar{\sigma}(t)=\left(\left[\left(A_{Q}\right)_{0}\right]^{-1} \circ \varphi_{0}^{-1}\right)(d l(\sigma(t))),
$$

we deduce that the curve $\Xi \circ \bar{\sigma}$ is the tangent lift of the curve $\mathcal{F}_{l} \circ \sigma$. 
Conversely, let $\bar{\sigma}: I \rightarrow S_{l}$ be a curve in $S_{l}$ such that

$$
(\Xi \circ \bar{\sigma})(t)=\frac{d}{d t} \gamma(t)
$$

where $\gamma: I \rightarrow T^{*} Q / G$ is a curve in $T^{*} Q / G$. Suppose that $\sigma: I \rightarrow T Q / G$ is the curve on $T Q / G$ associated with $\bar{\sigma}$, that is,

$$
\bar{\sigma}(t)=\left(\left[\left(A_{Q}\right)_{0}\right]^{-1} \circ \varphi_{0}^{-1}\right)(d l(\sigma(t))), \quad \forall t \in I .
$$

Then, using (6.11) and Lemma 6.3, it follows that

$$
\gamma(t)=\left(\tau_{T^{*} Q / G} \circ \Xi \circ \bar{\sigma}\right)(t)=\left(\tau_{T^{*} Q / G} \circ \Lambda\right)(d l(\sigma(t))) .
$$

From (6.6), (6.7) and (6.10), we obtain that

$$
\gamma(t)=\widetilde{\mathbf{v}^{*}}(d l(\sigma(t)))=\mathcal{F}_{l}(\sigma(t)) .
$$

Using (6.11) and (6.12) and Lemma 6.3, this proves that

$$
\frac{d}{d t}\left(\mathcal{F}_{l} \circ \sigma\right)(t)=\Lambda(d l(\sigma(t)))
$$

Therefore, $\sigma$ is a solution of the Lagrange-Poincaré equations for $L$.

Using this theorem, we obtain an intrinsic description of the Lagrange-Poincaré equations.

Corollary 6.5. Let $L: T Q \rightarrow \mathbb{R}$ be a $G$-invariant Lagrangian function, $l: T Q / G \rightarrow \mathbb{R}$ the reduced Lagrangian function and $\mathcal{F}_{l}: T Q / G \rightarrow T^{*} Q / G$ the Legendre transformation associated with l. A curve $\sigma: I \rightarrow T Q / G$ is a solution of the Lagrange-Poincaré equations for $L$ if, and only if, the image by $\Xi$ of the corresponding curve in $S_{l}$,

$$
t \rightarrow\left(\left[\left(A_{Q}\right)_{0}\right]^{-1} \circ \varphi_{0}^{-1}\right)(d l(\sigma(t)))
$$

is the tangent lift of the curve $\mathcal{F}_{l} \circ \sigma$.

The following diagram illustrates the previous situation

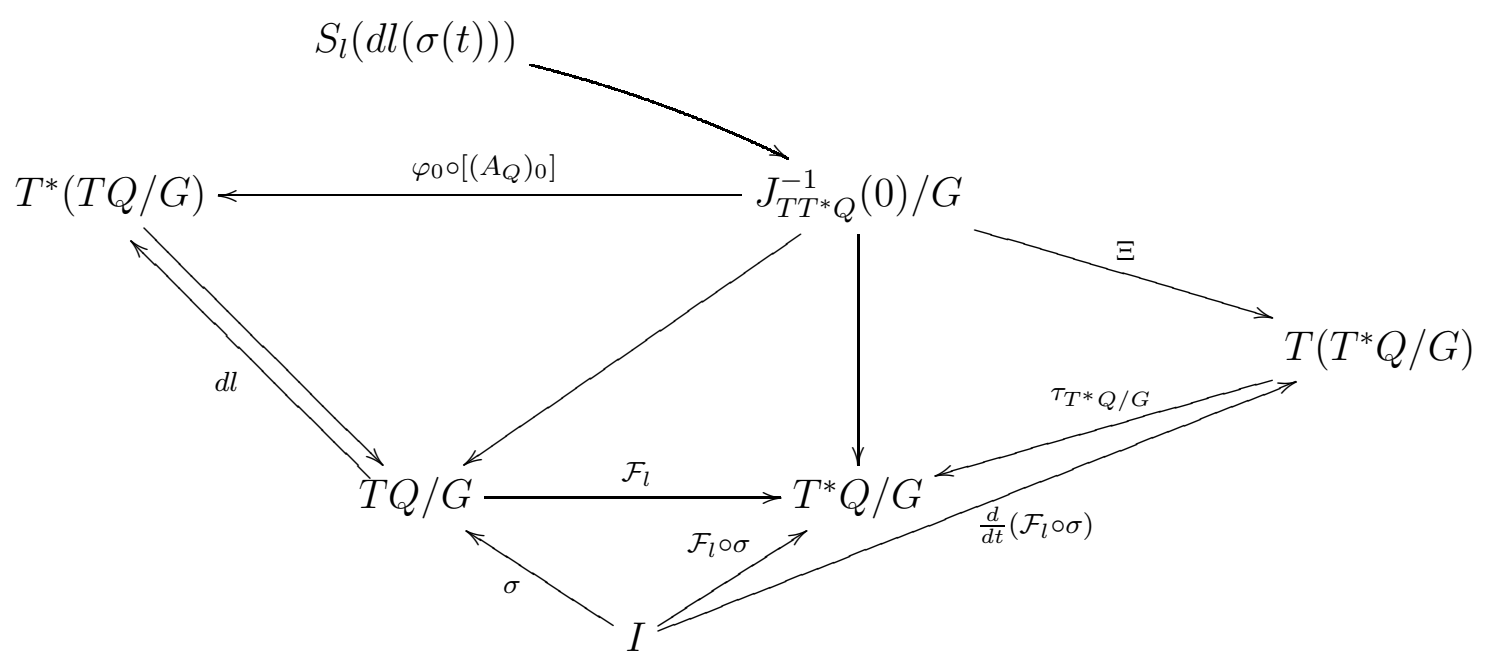

Remark 6.6. Using the above results and proceeding as in the Hamiltonian side (see Section (5) one may obtain a description of Euler-Poincaré equations (that is, LagrangePoincaré equations for the particular case when the configuration space is a Lie group) in terms of Lagrangian submanifolds of a symplectic manifold. 


\section{The equivalence Between the Reduced Lagrangian and Hamiltonian FORMALISM}

Consider the Liouville vector field $\Delta$ on $T Q$ given by $\Delta\left(v_{q}\right)=\left(v_{q}\right)_{v_{q}}^{v}$, for all $v_{q} \in T Q$. Suppose that $L: T Q \rightarrow \mathbb{R}$ is an invariant hyperregular Lagrangian and consider its $(G-$ invariant) energy $E_{L}=\Delta(L)-L$. The corresponding Hamiltonian function $H=E_{L} \circ \mathcal{F}_{L}^{-1}$ is also $G$-invariant. In many papers (see e.g. [16, 23, 24]) it has been shown that the submanifolds

$$
S_{L}=\left(A_{Q}\right)^{-1}(d L(T Q)) \text { and } S_{H}=\left(b_{\omega_{Q}}\right)^{-1}\left(d H\left(T^{*} Q\right)\right)
$$

coincide.

On the reduced level, by making use of (4.7), (6.8) and the previous fact, we obtain:

Theorem 7.1. Let $L$ be an hyperregular Lagrangian. If $l: T Q / G \rightarrow \mathbb{R}$ and $h: T^{*} Q / G \rightarrow$ $\mathbb{R}$ are the reduced Lagrangian and Hamiltonian functions and $S_{l}, S_{h}$ are the reduced Lagrangian submanifolds of the symplectic manifold $J_{T T^{*} Q}^{-1}(0) / G$, then $S_{l}=S_{h}$.

The reduced Tulczyjew triple below illustrates the situation.

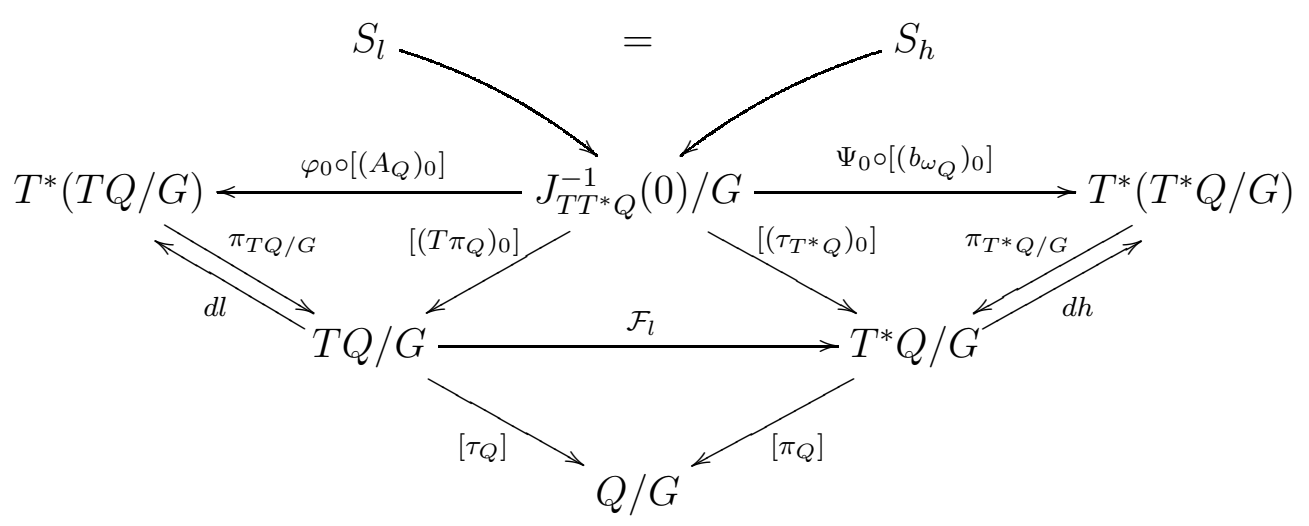

Here, $\left[\left(T \pi_{Q}\right)_{0}\right]: J_{T T^{*} Q}^{-1}(0) / G \rightarrow T Q / G$ (respectively, $\left.\left[\left(\tau_{T^{*} Q}\right)_{0}\right]: J_{T T^{*} Q}^{-1}(0) / G \rightarrow T^{*} Q / G\right)$ is the canonical projection induced by the vector bundle projection $\left(T \pi_{Q}\right)_{0}=\left(T \pi_{Q}\right)_{\mid J_{T T^{*} Q}^{-1}(0)}: J_{T T^{*} Q}^{-1}(0) \rightarrow T Q$ (respectively, $\left(\tau_{T^{*} Q}\right)_{0}=\left(\tau_{T^{*} Q}\right)_{\mid J_{T T^{*} Q}^{-1}(0)}:$ $\left.J_{T T^{*} Q}^{-1}(0) \rightarrow T^{*} Q\right)$.

\section{Conclusions And FUture WORK}

In this paper, we have described solutions of the Hamilton-Poincaré (respectively, LagrangePoincaré) equations in terms of Lagrangian submanifolds, thereby obtaining a reduced Tulczyjew triple entirely consisting of symplectic manifolds. In this section, we first want to place our results better in the context of the existing literature.

Note that, to obtain the dynamics, we have extensively made use of the map $\Xi$ that we had defined in expression (4.4). This map, actually, allows one to relate our triple with one that appeared in [9], associated with an arbitrary Lie algebroid $A$. If we apply the theory of [9] to the particular case when the Lie algebroid $A$ is the Atiyah algebroid 
$\left[\tau_{Q}\right]: T Q / G \rightarrow Q / G$ associated with the principal $G$-bundle $p_{Q}: Q \rightarrow Q / G$, then the resultant construction is the following diagram:

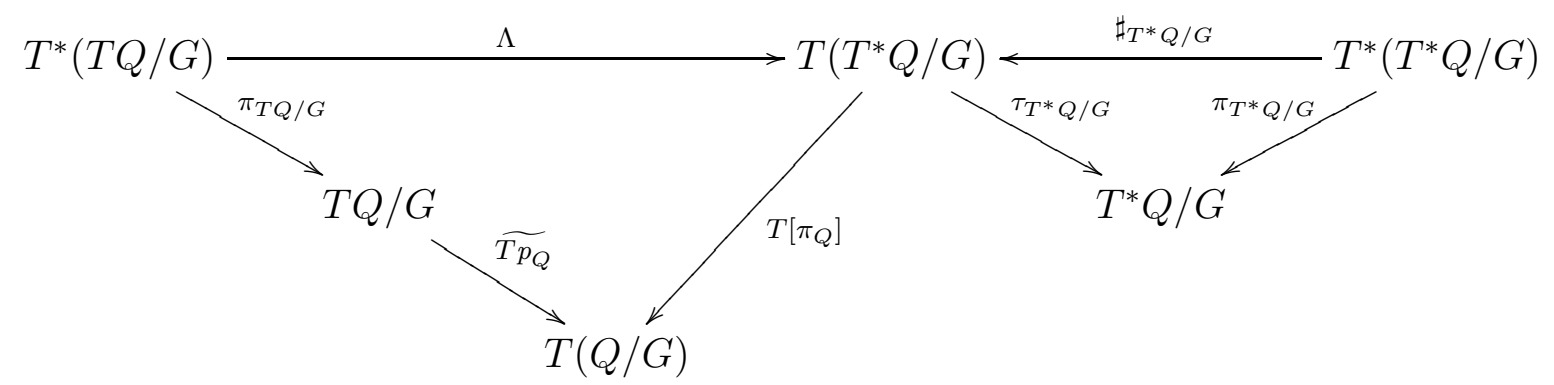

The space $T\left(T^{*} Q / G\right)$ is not, in general, a symplectic manifold. In fact, the complete lift of the linear Poisson structure on $T^{*} Q / G$ defines a (non symplectic) Poisson structure on $T\left(T^{*} Q / G\right)$. Thus, $T\left(T^{*} Q / G\right)$ is a Poisson manifold. Moreover, if $L: T Q \rightarrow \mathbb{R}$ is a $G$-invariant Lagrangian function and $l: T Q / G \rightarrow \mathbb{R}$ is the reduced Lagrangian function then $\left(\Lambda(d l(T Q / G))\right.$ is not, in general, a submanifold of $T\left(T^{*} Q / G\right)$. Note that $\Lambda$ is not, in general, a diffeomorphism.

Even though one may find in [9] an elegant way to describe the Lagrange-Poincaré and Hamilton-Poincaré equations, it seems natural to preserve the symplectic nature of the Tulczyjew triple after reduction. Since the morphism $\Xi: J_{T T^{*} Q}^{-1}(0) / G \rightarrow T\left(T^{*} Q / G\right)$ relates both Tulczyjew's triples, we have conveniently made use of it to relate the reduced dynamics, as described in [9], with the reduced Lagrangian submanifolds.

The following diagram illustrates the relation between the two triples.

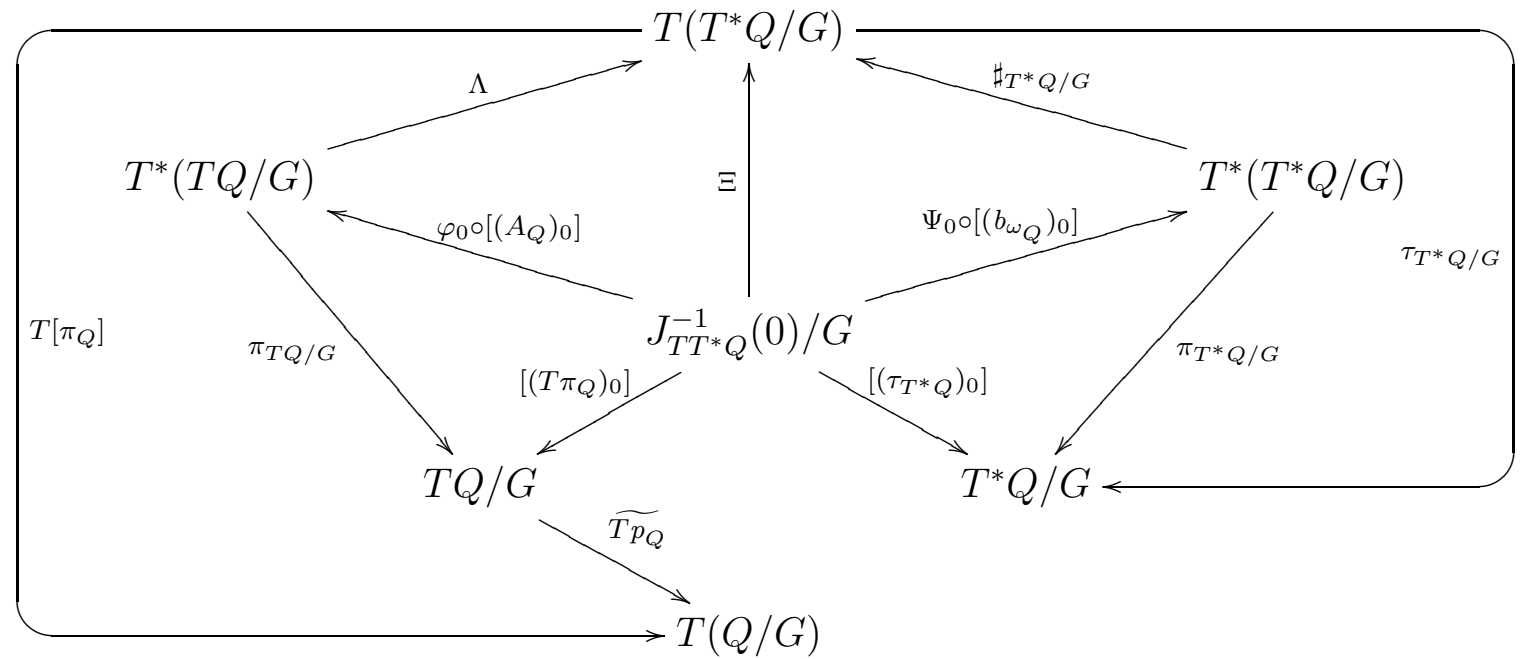

Using some results from [14, one may also deduce that solutions of the Hamilton-Poincaré (respectively, Lagrange-Poincaré) equations are in one-to-one correspondence with admissible curves in a Lagrangian submanifold of a symplectic Lie algebroid. We remark, however, that symplectic Lie algebroids cannot be considered genuine symplectic manifolds. It would therefore be of interest to compare these two different approaches.

We would also like to extend the results in this paper to classical field theories of first order. For this purpose, we can use the description in [2] (see also [6, 10, 15]) of these theories in terms of Lagrangian submanifolds of premultisymplectic manifolds and a suitable process of reduction of some special premultisymplectic manifolds. This will be the subject of a forthcoming paper (see [3]). 
The reduction methods we have applied so far mainly made use of the symmetry, and not so much of the fact that symmetry can sometimes be related to conservations laws (using e.g. Noether's theorem). Two reduction techniques which do make use of conservations laws are so-called Routh reduction (on the Lagrangian side) and cotangent bundle reduction (on the Hamiltonian side). In the papers [12, 13, 18] the close relation between these two theories have been brought in the spotlight, completely within a symplectic framework. It would therefore be of interest to investigate whether these two reduction theories can also be cast within a framework of a Tulczyjew triple.

\section{REFERENCES}

[1] R. Abraham, J. Marsden, Foundations of mechanics, Benjamin/Cummings Publishing Co., Inc., Advanced Book Program, Reading, Mass., (1978).

[2] C. M. Campos, E. Guzmán, J. C. Marrero, Classical field theories of first order and Lagrangian submanifolds of premultisymplectic manifolds, J. Geom. Mech. 4 (2012), no. 1, 1-26.

[3] S. Capriotti, J. C. Marrero, 2014 in preparation.

[4] H. Cendra, J.E. Marsden, T. Ratiu, Lagrangian reduction by stages, Mem. Amer. Math. Soc. 152 (2001), no. 722 .

[5] H. Cendra, J.E. Marsden, S. Pekarsky, T. Ratiu, Variational principles for Lie-Poisson and Hamilton-Poincaré equations, Mosc. Math. J. 3 (2003), no. 3, 833-867.

[6] K. Grabowska, The Tulczyjew triple for classical fields, J. Phys. A, 45 (2012), 145207-145242.

[7] K. Grabowska, J. Grabowski, Variational calculus with constraints on general algebroids, J. Phys. A 41 (2008), no. 17175204.

[8] K. Grabowska, J. Grabowski, Dirac algebroids in Lagrangian and Hamiltonian mechanics, J. Geom. Phys. 61 (2011), no. 11 2233-2253.

[9] K. Grabowska, P. Urbański, J. Grabowski, Geometrical mechanics on algebroids, Int. J. Geom. Methods Mod. Phys. 3 (2006), no. 3, 559-575.

[10] E. Guzman, J. C. Marrero, Time-dependent mechanics and Lagrangian submanifolds of presymplectic and Poisson manifolds, J. Phys. A, 43 (2010), 505201, 23 pp.

[11] K. Konieczna, P. Urbański, Double vector bundles and duality, Arch. Math. (Brno) 35 (1999), 59-95.

[12] B. Langerock, F. Cantrijn, J. Vankerschaver, Routhian reduction for quasi-invariant Lagrangians, J. Math. Phys. 51 (2010), no. 2, 022902, 20 pp.1089-7658.

[13] B. Langerock, T. Mestdag, J. Vankerschaver, Routh reduction by stages, Symmetry, Integrability and Geometry: Meth. Appl. (SIGMA) 7 (2011), 109, 31 pages.

[14] M. de León, J. C. Marrero, E. Martínez, Lagrangian submanifolds and dynamics on Lie algebroids, J. Phys. A 38 (2005), no. 24, R241-R308.

[15] M. de León, D. Martín de Diego, A. Santamaría-Merino, Tulczyjew triples and Lagrangian submanifolds in classical field theories, W. Sarlet, F. Cantrijn (eds.), Applied Differential Geometry and Mechanics. Gent, Academia Press (2003), 21-47.

[16] M. de León, P. R. Rodrigues, Methods of differential geometry in analytical mechanics, vol. 158 of North-Holland Mathematics Studies, North-Holland Publishing Co., Amsterdam, 1989.

[17] J.E. Marsden, G. Misiolek, J. P. Ortega, M. Perlmutter, T. Ratiu, Hamiltonian reduction by stages, Lecture Notes in Mathematics, 1913. Springer, Berlin, 2007.

[18] J.E. Marsden, T. Ratiu and J. Scheurle, Reduction theory and the Lagrange-Routh equations, J. Math. Phys. 41 (2000), 3379-3429.

[19] J.E. Marsden, T. Ratiu, Reduction of Poisson manifolds, Lett. Math. Phys., 41 (2000), 3379-3429.

[20] J.E. Marsden, A. Weinstein, Reduction of symplectic manifolds with symmetry, Rep. Mathematical Phys. 5(1974), no. 1, 121-130.

[21] T. Mestdag, A Lie algebroid approach to Lagrangian systems with symmetry, Differential Geometry and its Applications (Proc. Conf Prague, Czech Republic) ed J Bures et al (Prague: Matfyzpress), 523-535.

[22] T. Mestdag, M. Crampin, Invariant Lagrangians, mechanical connections and the Lagrange-Poincaré equations, J. Phys. A: Math. Theor. 41 (2008), 344015 (20pp).

[23] W. M. Tulczyjew, Les sous-variétés lagrangiennes et la dynamique hamiltonienne, C. R. Acad. Sci. Paris Sér. A-B, 283 (1976), A15-A18. 
[24] W. M. Tulczyjew, Les sous-variétés lagrangiennes et la dynamique lagrangienne, C. R. Acad. Sci. Paris Sér. A-B, 283 (1976), A675-A678.

[25] A. Weinstein, Symplectic geometry, Bull. Amer. Math. Soc. (N.S.) 5 (1981), no. 1, 1-13.

[26] H. Yoshimura, J.E. Marsden, Dirac structures in Lagrangian mechanics. I. Implicit Lagrangian systems, Journal of Geometry and Physics, 57 (2006), 133-156.

[27] H. Yoshimura, J.E. Marsden, Dirac cotangent bundle reduction, J. Geom. Mech., 1 (2009), 87-158.

Department of Mathematics, Ghent University, Krijgslaan 281, S22, B9000 Ghent (BelGIUM)

E-mail address: Eduardo.GToranoAndres@ugent.be

Ull-CSiC Geometría Diferencial y Mecánica Geométrica, Dept. Matemática Fundamental, Universidad de La laguna, Ull, Avda. Astrofísico Fco. Sánchez, 38206 la Laguna, TENERIFE (SPAin)

E-mail address: eguzman@ull.es

UlL-CSiC Geometría Diferencial y Mecánica Geométrica, Dept. Matemática Fundamental, Universidad de la laguna, Ull, Avda. Astrofísico Fco. Sánchez, 38206 la laguna, TENERIFe (Spain)

E-mail address: jcmarrer@ull.edu.es

Department of Mathematics, Ghent University, Krijgslaan 281, S22, B9000 Ghent (BelGIUM)

E-mail address: Tom.Mestdag@ugent.be 